\title{
The Influence of Wheelchair Users on Movement in a Bottleneck and a Corridor
}

\author{
Paul Geoerg $\mathbb{D}^{1},{ }^{1}$ Jette Schumann $\mathbb{D D}^{2},{ }^{2}$ Stefan Holl, ${ }^{2}$ and Anja Hofmann ${ }^{1}$ \\ ${ }^{1}$ Bundesanstalt für Materialforschung und -prüfung (BAM), Berlin, Germany \\ ${ }^{2}$ Forschungszentrum Jülich GmbH, Jülich, Germany \\ Correspondence should be addressed to Paul Geoerg; paul.geoerg@gmail.com
}

Received 1 February 2019; Revised 13 April 2019; Accepted 30 April 2019; Published 20 June 2019

Guest Editor: Milad Haghani

Copyright (C) 2019 Paul Geoerg et al. This is an open access article distributed under the Creative Commons Attribution License, which permits unrestricted use, distribution, and reproduction in any medium, provided the original work is properly cited.

Emergency exits as bottlenecks in escape routes are important for designing traffic facilities. Particularly, the capacity estimation is a crucial performance criterion for assessment of pedestrians' safety in built environments. For this reason, several studies were performed during the last decades which focus on the quantification of movement through corridors and bottlenecks. These studies were usually conducted with populations of homogeneous characteristics to reduce influencing variables and for reasons of practicability. Studies which consider heterogeneous characteristics in performance parameters are rarely available. In response and to reduce this lack of data a series of well-controlled large-scale movement studies considering pedestrians using different types of wheelchairs was carried out. As a result it is shown that the empirical relations $\bar{\rho}(\bar{v})$ and $\overline{J_{s}}(\bar{\rho})$ are strongly affected by the presence of participants with visible disabilities (such as wheelchair users). We observed an adaption of the overall movement speeds to the movement speeds of participants using a wheelchair, even for low densities and free flow scenarios. Flow and movement speed are in a complex relation and do not depend on density only. In our studies, the concept of specific flow fits for the nondisabled subpopulation but it is not valid for scenario considering wheelchair users in the population.

\section{Introduction}

Experimental investigations of pedestrian movements and dynamics have been widely improved during the last decades. Many studies under laboratory or field conditions have been performed to address movement characteristics, improve tracking methods, or investigate behavioural insights [1]. In particular fundamental diagrams are of strong interest to assess the performance of a facility. Frequently fundamental diagrams have been determined for experiments under laboratory conditions with a homogeneous (Homogeneous means in this case a comparative characteristic in attributes, e.g., similar range of age, soldiers, or students as participants, well-abled participants. In contrast, heterogeneous means a state of being diverse in skills and competencies relevant for movement (e.g., movement with vision loss, impeded movement, and using a wheelchair in one group)) configuration of the participating groups in terms of complexity of the type of movement (e.g., single-file movement [2-11], bidirectional movement [12-17], movement through corridors [5, 1822], considering bottleneck situations [5, 19, 20, 23-43], or multidirectional movement $[5,13,44-54])$.

The findings of this research were recently collected and comparatively prepared by extended reviews focussing on different flow types and geometries [55], empirical data collection with regard to complexity of movement [56], and movement dynamics [57]. In addition Haghani et al. [58] have compiled a detailed discussion on empirical methods on crowd behaviour and motion. Even these controlled boundary conditions result in significant differences in scope, shape, and characteristics [59]. It is debatable whether those data are still representative in terms of transferability to diverse, inhomogeneous, and more realistic populations. Given the fact that the demographic transformation process is already in progress ([60-63]) movement characteristics and functional relationships may be out of date [64]. To summarise, social-demographics in western societies are changing as the way of living and the kind of dwellings are. Particularly 
in the context of these developments and the associated lack of data, it is necessary to improve the understanding of movement behaviour of heterogeneous pedestrian groups.

Until now, only a few studies considered a more heterogeneous characterisation of participants. This challenge is addressed by a review of Geoerg et al. [65] who discussed the influence of reduced mobility on characteristics of movement. Daamen et al. [25, 26] have performed movement studies with bottleneck configuration considering participants with disabilities. A significant decrease of the bottleneck capacity for disabled participants is reported. The influence of age (elderly or younger subpopulations) on fundamental diagrams was investigated by $[25,26,66-73]$. A difference in characteristics of the fundamental diagrams was observed and stop-and-go waves are more probably to occur, if the population consists of a broader ageing range [70]. Contrary to this, Kolshevnikov et al. found that general characteristics of fundamental diagram considering elderly participants are comparable to younger populations [67]. An overall reduction of movement speed and flow for the elderly compared to younger adults in the studies under the same density situation is reported. The impact of vision loss, reduced visibility, or blindness in subpopulations was analysed by [74-78]. A slightly higher tendency to walk in groups or close to boundaries is reported by [76]. Furthermore, a reduction of flow rate and significantly more unstable flow conditions were reported by [77]. The importance of individual space requirements on flow and density was investigated by $[78,79]$ (presence of wheelchair users) or [80] (impact of luggage). An important influence by the presence of wheelchair users on flow characteristics is reported which is related to the required space of an individual. Additionally, research on several impacts like fatigue (decrease of movement speed with longer walking distance [81]), stress (higher urgency leads to a higher capacity [82]), or distraction (higher stopping duration with background music [83]) was published.

Concluding, little is known about the influence of heterogeneity on pedestrian movement characteristics which calls into the question of transferability for existing data to heterogeneous population settings. Therefore, this work is developed to analyse a series of well-controlled movement studies with focus on characteristics of pedestrians' movement and assessment of performance of a facility. The structure is organised as follows: in Section 2 the setting of the movement studies, the data extraction techniques, and the used calculation methods are introduced. The data analysis is subjected to Section 3. First, we present a comparison of unimpeded (unrestricted, free) movement speed in different subpopulations (Section 3.1). Second, the impact of the presence of wheelchair users in group movement is presented. Particularly the effect on fundamental diagrams $(\bar{v}(\bar{\rho}), \bar{J}(\bar{\rho})=\bar{\rho} \cdot \bar{v})$ is focused on (Section 3.2). Afterwards, the analysis of individual time gaps (Section 3.3) and the consequences on the specific flow (Section 3.4) are analysed. The conclusion will be made in the last section (Section 4).

\section{Methods}

2.1. Study Setup. The movement studies were conducted as a part of the interdisciplinary research project SiME (SiME is an acronym for the German meaning of "safety for all people" and is funded by the German Ministry of Education and Research) on two days in an industrial hall in Wermelskirchen-Dabringhausen (Germany) in June 2017. Twelve studies with more than 145 single runs and overall 252 participants with populations composed of people with and without disabilities were performed. In addition studies without any people with disabilities have been conducted.

Non-disabled participants (NDP) were recruited by public call while participants with disabilities (PWD) were recruited from a sheltered workshop. For defining the type of disability and to consider and select participants with disabilities, we used the Score RNA-approach, which is a method to weight potentially critical indicators for egress (for details we refer to $[84,85])$. The Score RNA considers disabilities in reception (blindness, deafness), perception (cognition), and realisation of movement (walking impairments, requirement on assistance devices) and in addition a cross-section variable (age) and evaluates the influence of the individual ability to evacuate autonomously.

The consideration of an appropriate proportion of people with disabilities in the overall population is oriented towards the prevalence of disability (approximately $10 \%$ in Germany [86], $15 \%$ for EU-27 [87], and $19 \%$ for the US [88]). In real scenarios pedestrians with disabilities do not occur in homogeneous groups. Their appearance in real situations is characterised by individual requirement on special aids, equipment, personal help, or assistance in the given situations. In this study, we focus on the impact of presence of wheelchair users as representatives of a heterogeneity (persona (for details of applying a persona we kindly refer to [89]) ), because the subpopulation deals with a variation in individual space requirements and movement characteristics (e.g., inertia, amplitude of swaying, and viewing height). Wheelchair users are therefore a necessary simplification for the design of persona "participants with disability."

We differentiate the population of a study into two subpopulations: on the one hand the participants with disabilities (disabled subpopulation) and on the other hand the participants without any disabilities (non-disabled subpopulation). In accordance with the reported ratio of inhabitants with disabilities, it has been tried to configure the populations in a similar ratio. In addition, we did reference studies for all geometrical settings with a reference population without any disabilities. The mean age of the disabled participants was $47.57 \pm 6.99$, and the mean age of nondisabled participants was $35.93 \pm 16.26$. The mean age of the reference population was $32.07 \pm 15.50$. The heights of all participants range from $1.45 \mathrm{~m}$ to $2.04 \mathrm{~m}$ with a mean of $1.74 \pm 0.1 \mathrm{~m}$.

Participation was absolutely voluntary for everybody and a cancellation of participation without any negative consequences was possible at any time. All participants have been paid $25 €$ per half a day of participation. Only anonymous data were used for the studies and the methodological design, data storage process, and the access authorisation for data 


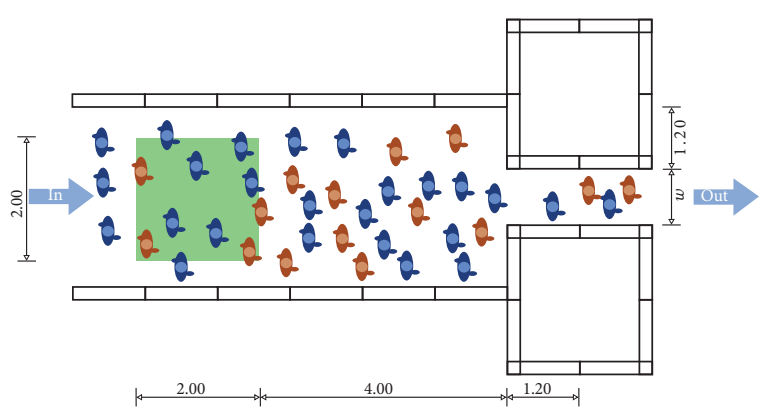

(a) Sketch of the bottleneck study

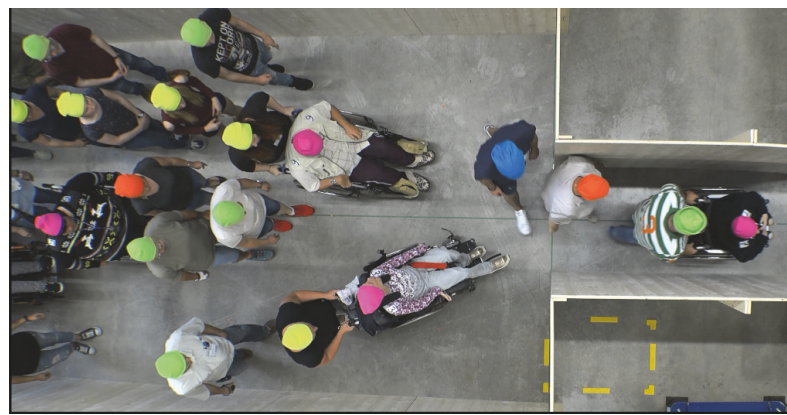

(c) Snapshot of the bottleneck study

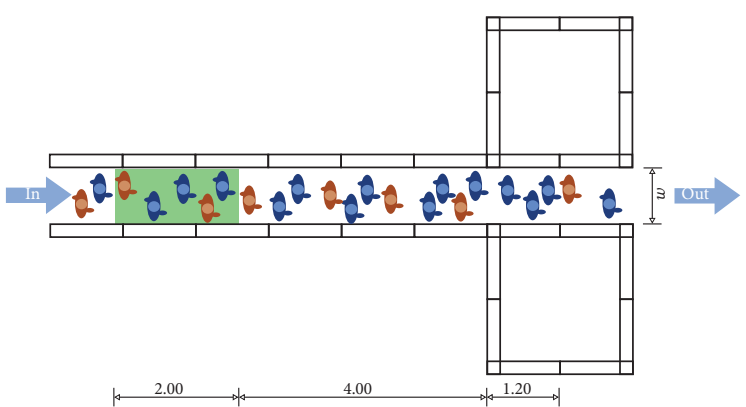

(b) Sketch of the corridor study

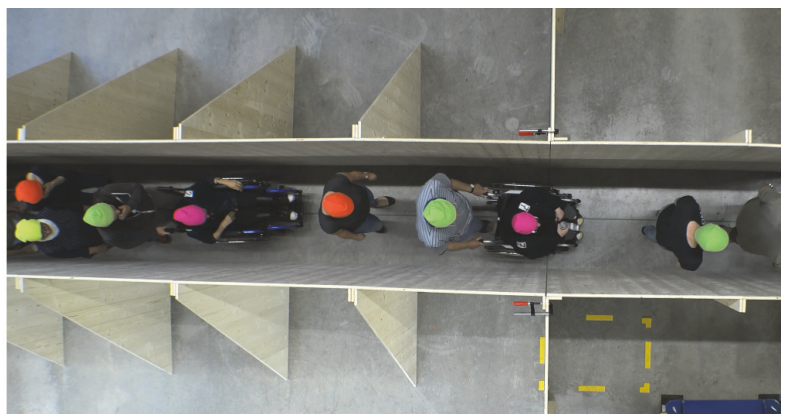

(d) Snapshot of the corridor study

FIGURE 1: Sketch of the study setup. The width $w$ is varied between 0.9 and $1.2 \mathrm{~m}$ in steps of $0.1 \mathrm{~m}$. Green rectangle: measurement area.

were approved by the ethics committee of the Bergische Universität Wuppertal. No ethical concerns were mentioned.

The aim of the studies was to investigate the impact of presence of wheelchair users on performance criteria for movement in built environments under laboratory conditions. Following the classification of complex movements by Shi et al. [56], we focused on investigations of internal driven movements in multilane pedestrian traffic. This includes effects like self-slowing, grouping queuing and is represented in corridors and egress situations (bottlenecks). To control the possibility of overtaking (corridor) and the density/queuing in front of the bottleneck (as a representative for a limited flow), the passage width $w$ was varied from $w=(0.9,1.0,1.1,1.2) \mathrm{m}$. The minimum width for doors in public accessible buildings in Germany is $0.9 \mathrm{~m}$, which is the reason to set the minimum width to a clear width of $0.9 \mathrm{~m}$ [90]. Expecting approximately 120 participants during the studies, it was estimated that a steady state concerning densities of approximately $3 \mathrm{~m}^{-2}$ will hardly be reached at large widths. Thus, the width was increased in steps of $0.1 \mathrm{~m}$ up to a maximum width of $1.20 \mathrm{~m}$ (which is in line with [37]). The length of the bottleneck was constant $(l=2.4 \mathrm{~m}$, see Figure 1). The geometry was built from wooden three-layer panels with a height of $2.0 \mathrm{~m}$. A waiting zone of $\approx 30 \mathrm{~m}^{2}$ was located at $x=[-18,-12]$ with an initial density of $\approx 3.0 \mathrm{~m}^{-2}$. The distance was meant to buffer starting conditions and minimize effects of entering the geometry. Wheelchair users were placed randomly over the entire space. The participants were advised to move through the bottleneck without haste. It was emphasized not to push and to walk with the preferred movement speed. A run was started on instruction of the experiment leader. When a participant leaves the geometry, he or she returns to the waiting zone for the next run. In order to avoid outliers, every run was repeated twice. Table 1 summarises the configuration and characteristics of the analysed runs in this article. The characteristics were measured in a measurement area of $4.0 \mathrm{~m}^{2}$ located four meters in front of the bottleneck entrance (see Figures 2 and 4 ).

Every experimental design is characterized by research pragmatic decisions, priorities, and a focus on complexity reduction. The authors are aware that this results in limitations for transferability to real scenarios. In particular, such narrow and long corridors are rarely found in real geometries. The decision for the geometric boundary conditions was made against the background of investigating the transition from single-file movement to group movement (corridor). On the other hand, bottlenecks are defined by their temporal and/or local limitation. The influence of the output from the bottleneck and the empirically observed capacity-increasing effect of very short bottlenecks [32] should be excluded to reduce complexity (bottleneck situation). In summary, the geometric configuration does not represent a real situation in a building. It focuses on creating the spatial boundary conditions: preventing and later enabling lane formation (corridor) and regulating the outflow given constant inflow (bottleneck).

2.2. Data Extraction. The passageway through the study setting was captured by nine high-definition cameras attached to the ceiling of the hall (height $\approx 6.34 \mathrm{~m}$ ). Each participant wore a coloured cap according to their individual body height. We used the PeTrack-Framework to determine the 
TABLE 1: Controlled boundary conditions and characteristic of each run. Note that some participants have to rest after a run due to their disability and that volunteers without tasks participated run-wise.

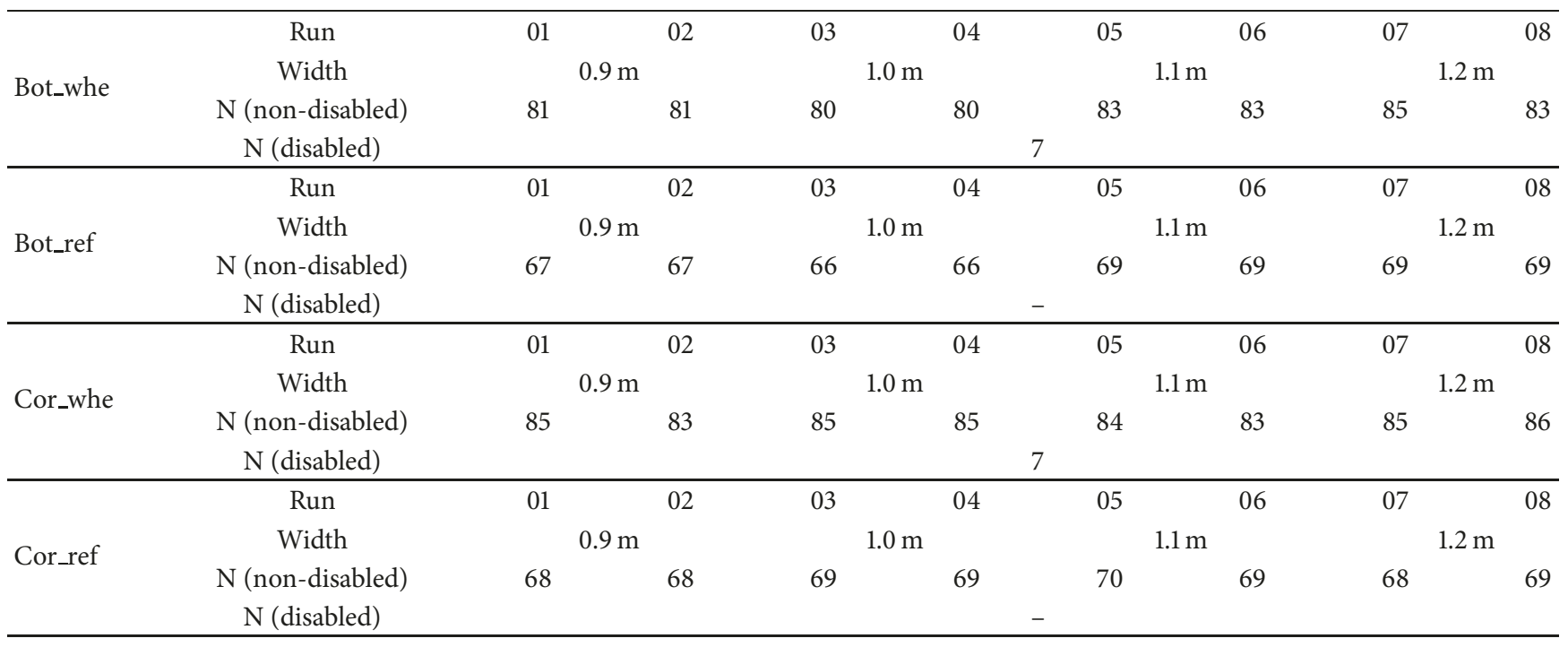

automatic extraction of positions at every time step (frame) from the video recordings [91]. PeTrack detects the center of the coloured cap and tracks the position in the next time step. The transformation from pixel coordinates into physical (world) coordinates was autocorrected by taking the height according to the colour of the caps into account [92]. We assume a coherent area of pixels in the middle of the coloured caps as body centre of a participant, so the resulting trajectories represent positions of the head projected on the ground. All resulting trajectories were checked and corrected manually. The trajectories are precise with a maximum error of $\approx 0.092 \mathrm{~m}$ in the perspective of the centred camera (for details of the error calculation we refer to [92]). Figure 2 displays the trajectories corresponding to the runs Bot_whe_01, Bot_ref_01, Cor_whe_01, and Cor_ref_01. Participants with disabilities (in this case using a wheelchair) are coloured in orange. For summarised configuration details see Table 1.

2.3. Calculation of Characteristics. Calculation of characteristics is separated into different approaches: (a) values of a fixed time interval (space-time-mean, according to [13]) to calculate the fundamental diagram, (b) the flow $J$ through the bottleneck at a fixed distance (time-mean, according to method A in [22]), and (c) values based on the Voronoi method (space-mean, according to [93]) to analyse the instantaneous movement speed along a given trajectory.

Method (a). We used a method to calculate the characteristics of the fundamental diagram which is able to provide mean values for density and movement speed at the same space and time. The mean density $\bar{\rho}(A, \Delta t)$ is the individual share of a participant $i$ at the total density in context of their effective time $\left[t_{0}, t_{1}\right]$ in the measurement surface $A$ during the measurement interval $\Delta t_{i}(A)$ and the length of the time interval (see (1)). The density within the measurement area is defined as the sum of the individual shares of the density [13].

$$
\bar{\rho}(A, \Delta t)=\sum_{i=1}^{n} \frac{t_{i}(A)}{\Delta t} \cdot \frac{1}{A}
$$

The mean movement speed $\bar{v}(A, \Delta t)$ is defined as the ratio of the sum of all covered distances $e_{i}(\Delta t)$ during the sampling interval $\left[t_{0}, t_{1}\right]$ to the sum of the time spent $\Delta t_{i}(A)$ on the measurement area $A$ (see (2)).

$$
\bar{v}(A, \Delta t)=\frac{\sum_{i=1}^{n} e_{i}(\Delta t)}{\sum_{i=1}^{n} \Delta t(A)}
$$

Method (b). The flow rate $J$ based on the time gaps of the participants is similar to the number of participants crossing the fixed location at $x=0$ [57]. Therefore, the time gaps $\Delta t_{i}=$ $t_{i+1}-t_{i}$ are calculated between the consecutive participants $i$ and $i+1$. The sum of the individual time gaps is directly related to the flow (see (3) and (4)).

$$
\begin{aligned}
J & =\frac{1}{\Delta t_{i}} \quad \text { with } \Delta t_{i}=\frac{1}{N} \sum_{i=1}^{N}\left(t_{i+1}-t_{i}\right) \\
& =\frac{N}{\sum_{i=1}^{N}\left(t_{i+1}-t_{i}\right)}
\end{aligned}
$$

The specific flow $J_{s}$ giving the flow per unit width is defined as the normalised flow $J$ related to the width (see (5)).

$$
J_{s}=\frac{J}{w}
$$

Method (c). The Voronoi tessellation assigns an individual required space $A_{i}(t)$ to each person (called Voronoi cell) which includes all coordinates of the Euclidean plane closer to 


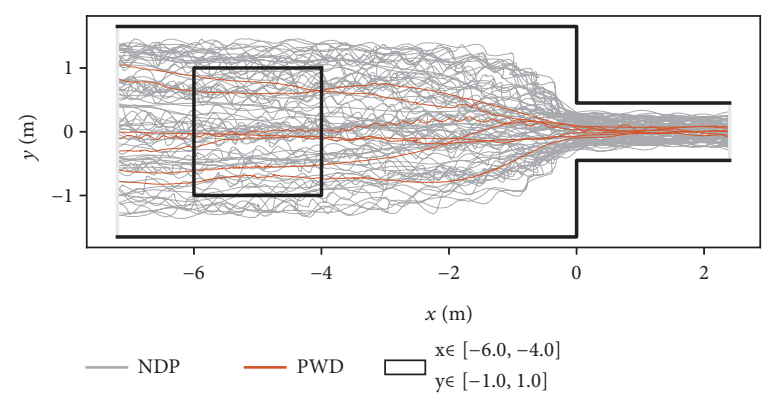

(a) Trajectories for run Bot_whe_01 with $\approx 10 \%$ participants using wheelchairs

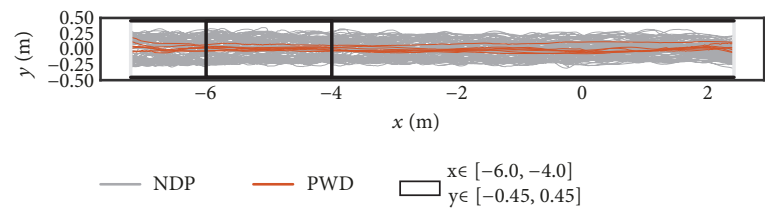

(c) Trajectories for run Cor_whe_01 with $\approx 10 \%$ participants using wheelchairs

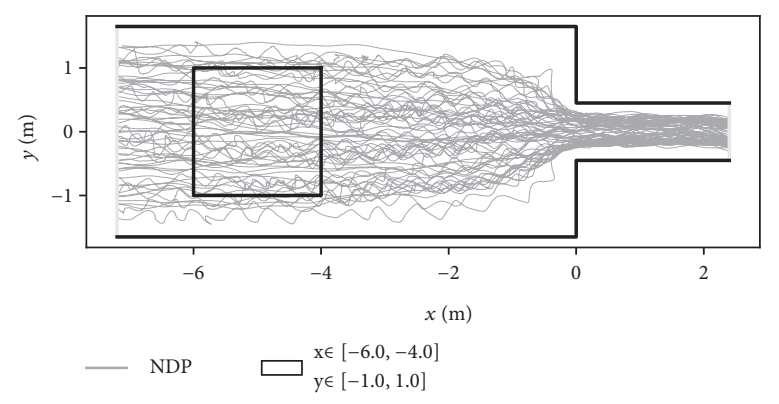

(b) Trajectories for run Bot_ref_01 without participants using wheelchairs

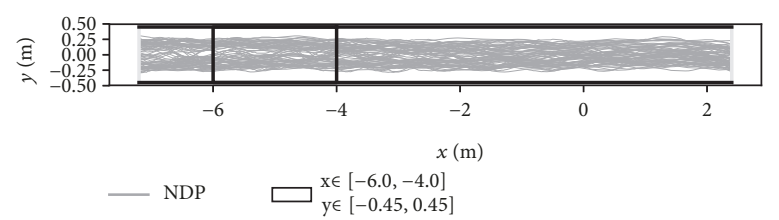

(d) Trajectories for run Cor_ref_01 without participants using wheelchairs

FIGURE 2: Individual trajectories of movement through a bottleneck and a corridor with a width of 0.9 m considering participants using a wheelchair (left) and without participants with disabilities (right). Participants using a wheelchair are coloured in orange. Black rectangles represent the measurement surface (coordinates are given in the legend).

TABLE 2: Unimpeded movement speeds in $\mathrm{ms}^{-1}$ for different subpopulations. For a comprehensive description of the characteristics we kindly refer to [84]. Data is presented in the following format: mean [standard deviation, minimum - maximum].

\begin{tabular}{lccrr}
\hline Study & $\mathrm{N}(\mathrm{PWD})$ & $v_{0}(\mathrm{PWD}) / \mathrm{ms}^{-1}$ & $\mathrm{~N}(\mathrm{NDP})$ & $v_{0}(\mathrm{NDP}) / \mathrm{ms}^{-1}$ \\
\hline Elderly $^{\mathrm{a}}$ & 4 & $1.30[0.20,1.08-1.58]$ & 81 & $1.42[0.14,1.02-1.75]$ \\
Cognition $^{\mathrm{b}}$ & 9 & $1.20[0.09,1.04-1.31]$ & 78 & $1.47[0.14,1.09-1.81]$ \\
Wheelchair $^{\mathrm{c}}$ & 7 & $0.96[0.35,0.45-1.41]$ & 70 & $1.45[0.17,0.63-1.82]$ \\
Walk $^{\mathrm{c}}$ & 5 & $1.28[0.12,1.07-1.41]$ & 74 & $1.44[0.17,0.88-2.08]$ \\
Mixed $^{\mathrm{d}}$ & 5 & $0.71[0.26,0.41-1.15]$ & 71 & $1.43[0.20,0.39-1.87]$ \\
Hetero $^{\mathrm{e}}$ & 12 & $1.33[0.29,0.29-1.65]$ & 68 & $1.47[0.18,0.65-1.89]$ \\
Reference & & & $1.47[0.17,1.08-1.86]$ \\
\hline
\end{tabular}

${ }^{a}$ Participants with an age $\geq 60$ years without any other disability.

${ }^{\mathrm{b}}$ Participants with a cognition impairment (MELBA SL Score $\leq 0.5$. MELBA-SL is a profile to assess the work participation of people with impairments in Germany (see [96], available only in German; for an English summary, see [97]). MELBA-SL measures and classifies 29 different characteristics to evaluate the work ability of impaired persons. The MELBA-approach evaluates individual abilities with regard to requirements to a concrete work. Attributes are rated in MELBA are for instance attention, resolving problems, degree of empowerment, carefulness, reading, writing and so on.

${ }^{c}$ Participants with marking in the severely handicapped pass.

${ }^{\mathrm{d}}$ Mixed participants with different single disabilities.

${ }^{\mathrm{e}}$ Mixed participants with different multiple disabilities.

the participant $i$ than to the neighbours $([8,93,94])$. Density inside a Voronoi cell of a participant is equal to the reciprocal of its area $\left(\rho_{x, y, t}(i)=1 / A_{i}\right)$. The Voronoi density and velocity for the measurement area are then defined as

$$
\begin{aligned}
& \rho_{v}(x, y, t, \Delta x, \Delta y)=\frac{\iint \rho(x, y, t) d x d y}{\Delta x \cdot \Delta y} \\
& \text { with } \Delta y=w \\
& v_{v}(x, y, t, \Delta x, \Delta y)=\frac{\iint v(x, y, t) d x d y}{\Delta x \cdot \Delta y}
\end{aligned}
$$

\section{Analysis and Results}

3.1. Unimpeded Movement Speed. The unimpeded (free) movement speed $v_{0}$ for each participant was measured before starting the studies (Table 2). The participants were ask to move alone through the bottleneck setting with a width of $w=0.9 \mathrm{~m}$. The movement start of each individual was controlled and we considered a distance of $\approx 5 \mathrm{~s}$ between each start. This leads to an interpersonal distance of $\approx 6 \mathrm{~m}$, if we suppose a free movement speed of $\approx 1.2 \mathrm{~ms}^{-1}$.

Overall, the observed unimpeded movement speeds of nondisabled participants are comparable with literature findings (see [1]), which indicates a working experimental setting. On the other hand, unimpeded movement speeds of the 


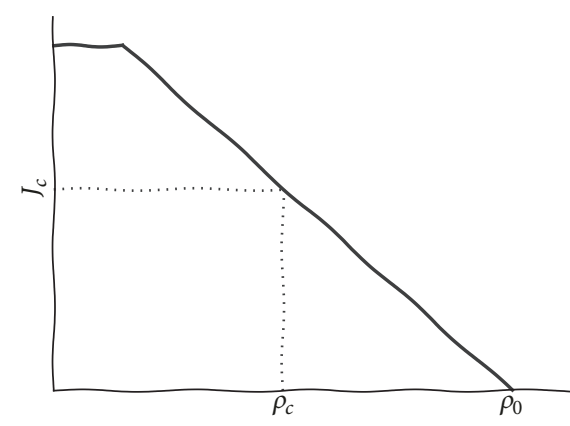

(a) $v(\rho)$

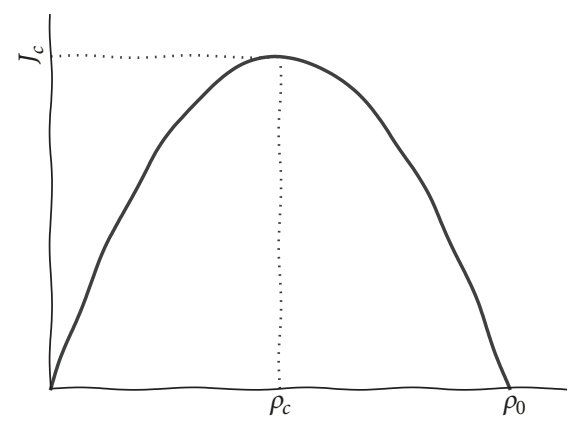

(b) $J_{s}(\rho)$

FIGURE 3: Schematic representation of the fundamental diagram $v(\rho)$ and $J_{s}(\rho)$ (visualisation according to [95]).

participants with disabilities depend strongly on individual characteristics such as the kind of movement ability which leads to high standard deviations. For this reason, it is difficult to derive general statements about movement characterisation [84]. Due to the small sample size for the disabled participants, the results represent in particular the relation of the individuals in this study and the results should be verified in further experiments.

3.2. Influence on the Fundamental Diagram. In general, a fundamental diagram describes the relation between density and movement speed or density and flow. It indicates the importance for calculation methods for dimensioning pedestrian facilities [57]. Quantities for characterisation of the fundamental diagrams are the capacity $J_{s, \max }$, the density $\rho_{c}$ where $J_{s, \max }$ is reached, and $\rho_{0}$ where the overcrowding results in impossibility of movement [39]. A fundamental diagram consists of three branches [95] (see Figure 3):

(i) a free-flow branch at low density, where the flow increases with density and the movement speeds are similar to the desired (unimpeded) movement speeds

(ii) a branch where interactions between pedestrians occur with needs for changes of velocity and directions but still a stable flow

(iii) a congested branch at higher densities where the flow decreases with increasing density due to the distance to neighbours and which in consequence leads to the formation of jams

The obtained trajectory data enable analysing the movement through the geometries in relation of space and time. Figure 4 shows the movement of each participant through the bottleneck with different widths. Sequences of the trajectories with a slow instantaneous movement speed $\left(\leq 0.1 \mathrm{~ms}^{-1}\right.$ as calculated by method (c), described in Section 2.3) are coloured in red. Data for the subpopulation with wheelchair users (left) and the reference population (right) are shown.

It can be seen that the movement through the bottleneck for the reference population is uniform and homogeneous for all widths. All participants overcame the same distances by a similar time and without overtaking and clogging effects. The overall passage time decreases with increasing bottleneck width (from top to down). In contrast, the data for a population with wheelchair users are presented on the left side in Figure 4. Significant changes of uniformity in movement were observed in front of the bottleneck. Waiting phases (lower increase of the trajectory in y-direction) and overtaking situations (crossing of trajectories) result in an inhomogeneous movement characteristic (situational change of instantaneous movement speed). This phenomenon is called shockwave and it is well known from incidents or bottlenecks on road traffic and pedestrian dynamics [98, 99]. Shockwaves occur in case of transition between two traffic states (e.g., free flow to congested) [100]. The individual (Voronoi) movement speed is depending on time and $\mathrm{x}$ position and is presented in two colours in Figure 4:

$$
\begin{aligned}
& \text { colour }=\text { red }, \quad \text { if } v_{v} \leq 0.1 \mathrm{~ms}^{-1} \\
& \text { colour }=\text { grey, } \text { otherwise }
\end{aligned}
$$

They occur more likely in case of presence of wheelchair users and propagate with certain speed upwards the flow $\left(v_{\text {jam }}\right)$ and clearly related to the entrance of the person with disability at the bottleneck.

We focus on the fundamental diagrams to analyse the characteristics of movement depending on population characteristics (Figures 6 and 7). The presented fundamental diagrams show the mean values and the standard deviations over all runs (and different widths). The relation between movement speed and density and flow and density is based on time-space-mean calculations (method (a), described in Section 2.3) introduced by Eddie [101] and generalised by [13]. A time interval of $\Delta t=2 \mathrm{~s}$ (which is corresponding to 50 frames) was used to calculate the moving averages of the characteristics.

To determine the fundamental diagram only data from stable conditions are used (see the grey coloured area in Figure 5). This is particularly important since pedestrian dynamics experiments usually have significantly shorter running times due to limited personnel and financial factors which is unlike in field studies of vehicle traffic. While in field studies of car traffic periods of three to five minutes are not uncommon for the determination of moving averages [102], pedestrian experiments are often shorter than two minutes due to limited resources. Thus, a significant part of 


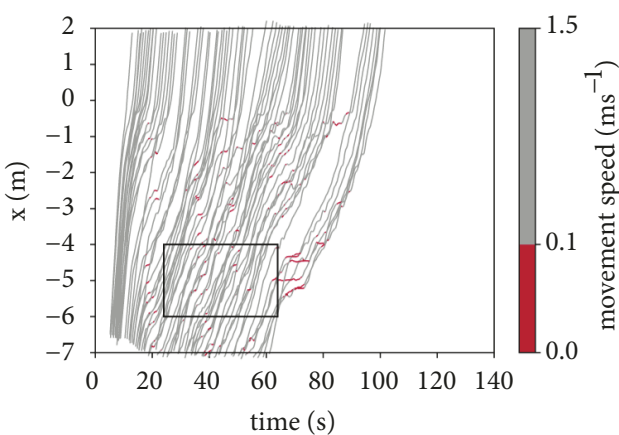

(a) Bot_whe_01 $(w=0.9 \mathrm{~m})$

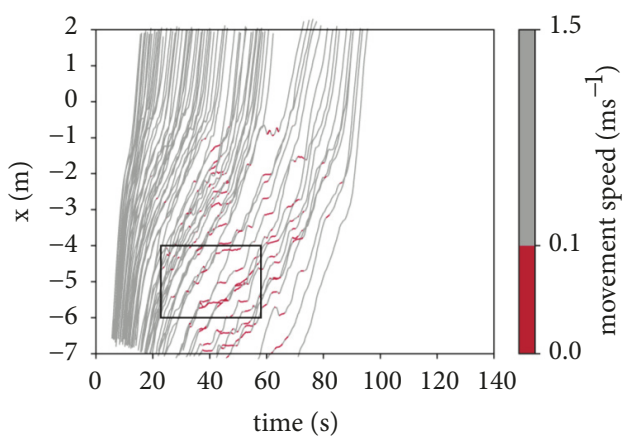

(c) Bot_whe_03 $(w=1.0 \mathrm{~m})$

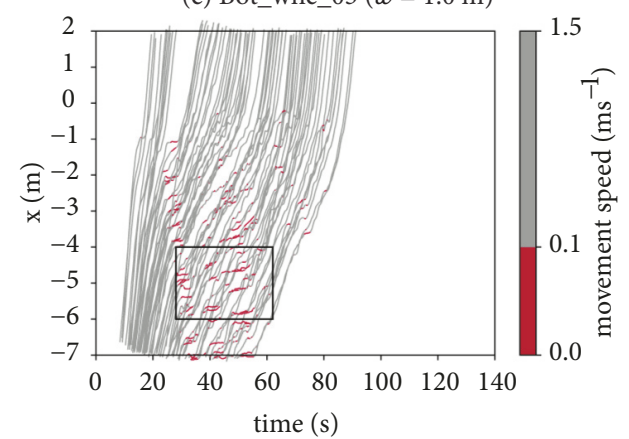

(e) Bot_whe_05 $(w=1.1 \mathrm{~m})$

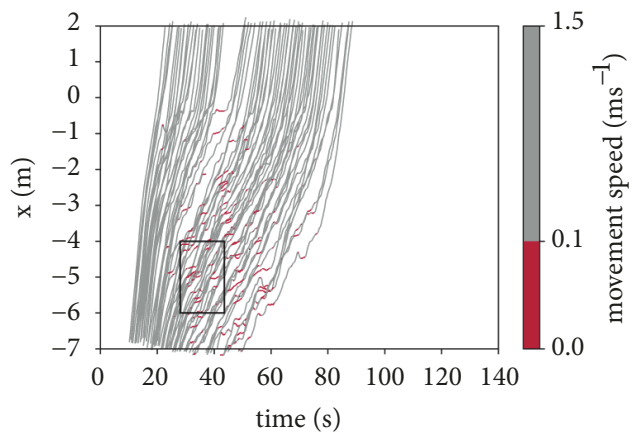

(g) Bot_whe_07 ( $w=1.2 \mathrm{~m})$

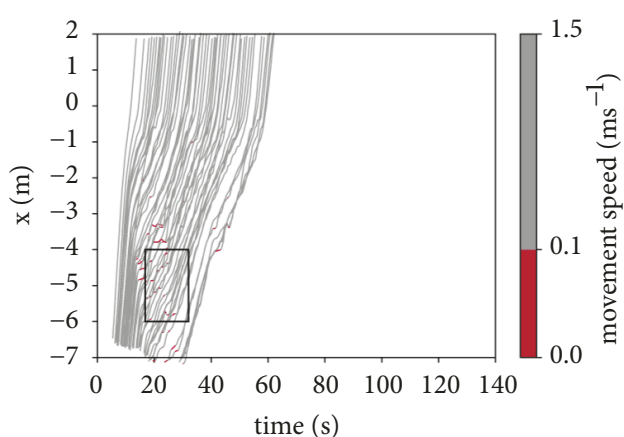

(b) Bot_ref_01 $(w=0.9 \mathrm{~m})$

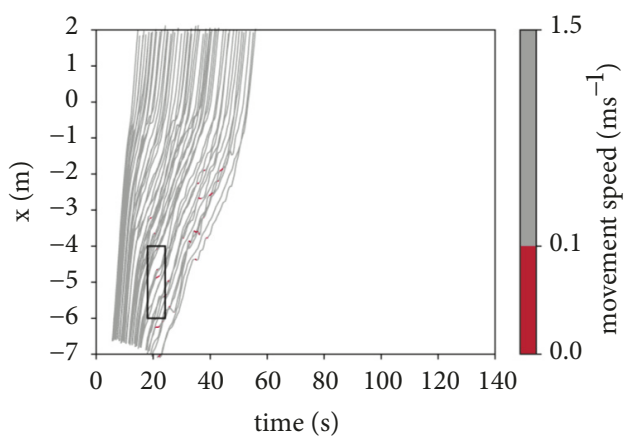

(d) Bot_ref_03 $(w=1.0 \mathrm{~m})$

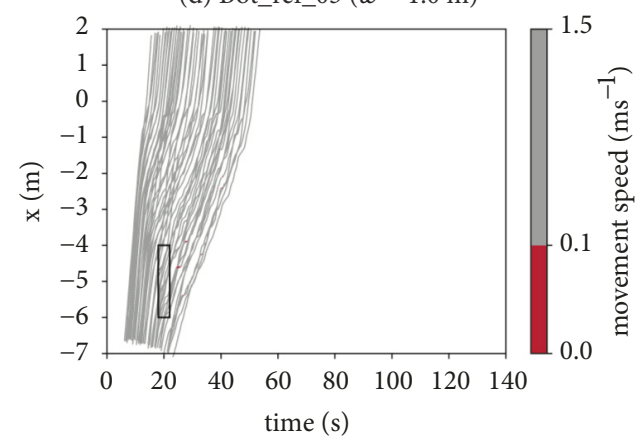

(f) Bot_ref_05 $(w=1.1 \mathrm{~m})$

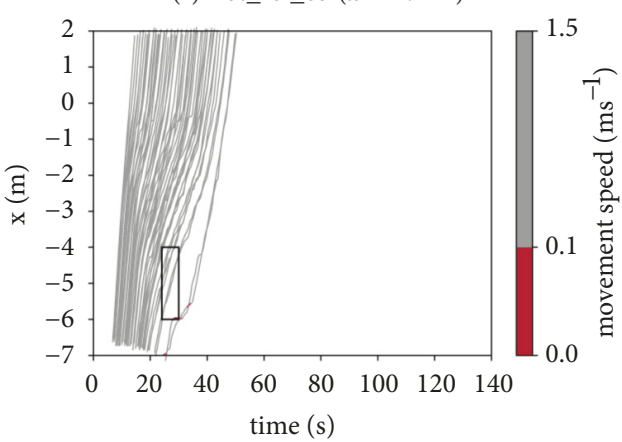

(h) Bot_ref_07 $(w=1.2 \mathrm{~m})$

FIGURE 4: Time-space plots of movement through a bottleneck with different widths considering participants using a wheelchair (left) and without participants with disabilities (right). Black rectangle: measurement surface in space and time (steady states). The threshold for visualisation was defined by $0.1 \mathrm{~ms}^{-1}$.

the time period depends on (random distributed) start and end conditions. Since a central objective of the investigation was the analysis of the influence of heterogeneous movement properties on the stability of the values of interest, an automatic detection, as, for example, the Cumulative Sum Control
Chart algorithm [31], did not lead to the consideration of the desired time periods. For this reason steady states are defined by the relative stable condition of the time development [30]. In this work, the steady state conditions were detected and manually selected by analysing the time series for movement 

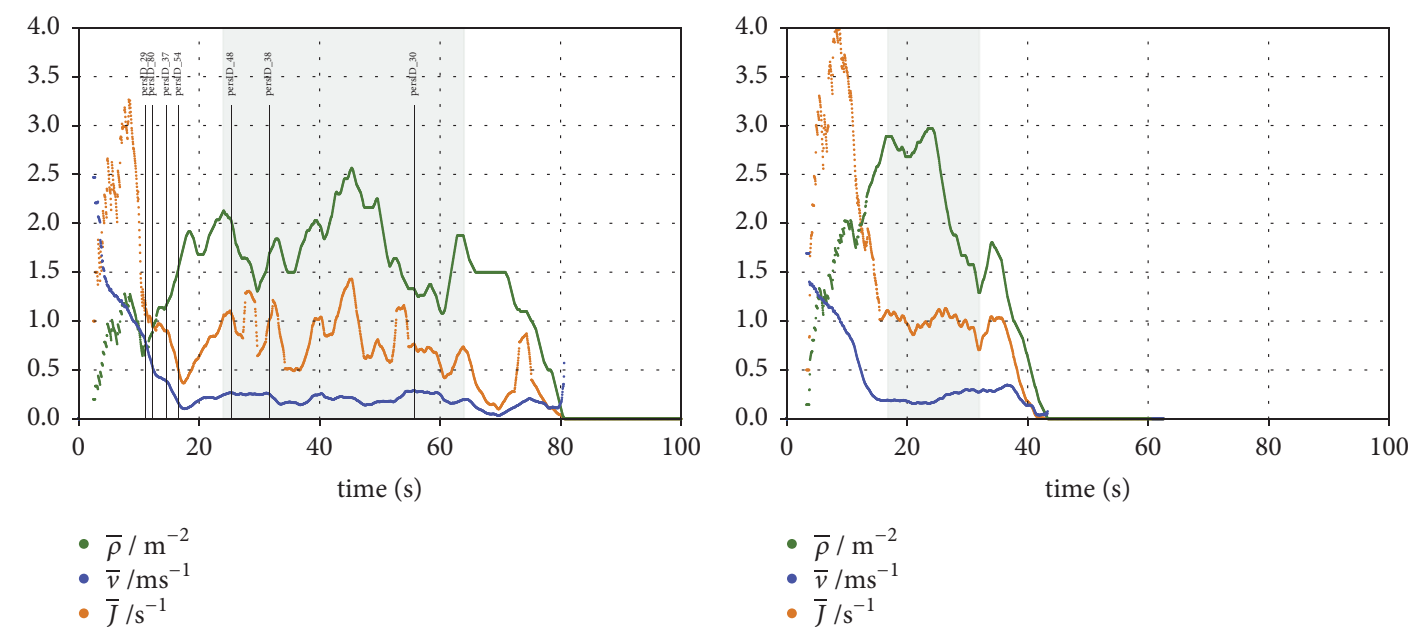

(a) Time development with wheelchair subpopulation (Bot_whe_01)

(b) Time development with reference population (Bot_ref_01)

FIGURE 5: Time series of $\bar{\rho}(t), \bar{v}(t)$, and $\bar{J}(t)$ for a bottleneck with a subpopulation of wheelchair users (left) and a reference population (right) in a width of $0.9 \mathrm{~m}$ and calculated by method (a). The manually detected steady state is coloured in light grey. The entrance of participants in wheelchairs into the measurement surface is emphasised by the vertical lines.

speed, density, and flow (in accordance with the work by $[10,13,30,52,94,103])$.

In result the shape of the measurement surface depends on space and time. In constant space-dimension we took the measurement in a rectangular area in a distance of $4 \mathrm{~m}$ to the bottleneck entrance (Figures 1 and 2). In variable time-dimension, the spread of the measurement surface was adapted by the steady state definition (see black rectangles in Figure 4).

The steady state for the configuration with wheelchair users takes the significant fluctuations and multiple local maxima in density and flow (caused by the inhomogeneous movement in space and time) into account. For the bottleneck configuration the density increases within $25 \mathrm{~s}$ from initial density up to a local maximum for bottleneck and remains steady (but fluctuating) for $\approx 40 \mathrm{~s}$ (Figure 5(a)). In consequence, the process of obtaining the fundamental diagram is in balances between considering the data of disabled participants (fluctuating data) and the need for stable conditions (steady states). In contrast, characteristics for the reference run without any wheelchair users (Figure 5(b)) are marked by a strong increase of density to a plateau of $2.8 \mathrm{~m}^{-2}$ which is only steady for $\approx 10 \mathrm{~s}$.

Because of the limited number of participants, a polite and considerate behaviour, and the motivation of the participants, we only observed mean density values between $1.0 \mathrm{~m}^{-2}$ and $3.0 \mathrm{~m}^{-2}$. As a consequence, we did not reach the congested state of the fundamental diagrams in our studies (see Figure 3 ). The maximum density of $\approx 3 \mathrm{~m}^{-2}$ without participants in wheelchairs and $\approx 3.8 \mathrm{~m}^{-2}$ in the bottleneck studies was observed (Figure 6). Also the maximum density for the corridor studies differs for the presence of wheelchair users: if wheelchair users attend, the maximum density $\bar{\rho}_{\max }$ is $\approx 3 \mathrm{~m}^{-2}$. In the reference studies without presence of wheelchair users, we observed maximum densities of $\bar{\rho}_{\text {max }} \approx$ $2.3 \mathrm{~m}^{-2}$. Due to the lower movement ability, flexibility, and higher requirement in (static) space, participants in wheelchairs reside more likely in lower density regions than the participants without disabilities.

It is noticeable that the observed mean movement speed is remarkably slow and-in case of the bottleneck situation-independent of the density (Figure 6). This is in contrast to the expected behaviour and to data from previous studies with homogeneous populations (e.g., $[2,37,57]$ ) and the classic understanding of the fundamental diagram. However, movement speed in the corridor shows a slight dependency on the density for both population settings (Figure 7). The variation in observed movement speeds is much higher in case of presence of wheelchair users.

A dependency of the (hydrodynamic) flow on density was observed for both geometries and is independent of the population configuration. In both presented studies, the capacity $J_{s, \max }$ was presented where $\bar{\rho}_{c}$ was not fully reached. In case of the bottleneck geometry, the flow slightly increases with the density but reaches a plateau of $\approx 1.1 \mathrm{~m}^{-1} \mathrm{~s}^{-1}$ in case of presence of wheelchair users. A monotonically increased flow without reaching the capacity limit $\left(J_{s, \text { max }}\right)$ was observed for the reference population. The flow increases monotonously with the density in the corridor studies and reaches the capacity where the flow will turn into congested state (and decrease) at $\bar{\rho} \approx 2.5 \mathrm{~m}^{-2}$ in case of the participation of wheelchair users or $\bar{\rho} \approx 2.0 \mathrm{~m}^{-2}$ otherwise. This is because of the low density region of the studies and because of an anticipation of movement speed to the slower wheelchair users (compare similar colours which represent the instantaneous movement speed of each attendee in Figure 4). Because of the latter and due to width of maximum $1.2 \mathrm{~m}$ overtaking of wheelchair users was not possible which has as consequence 


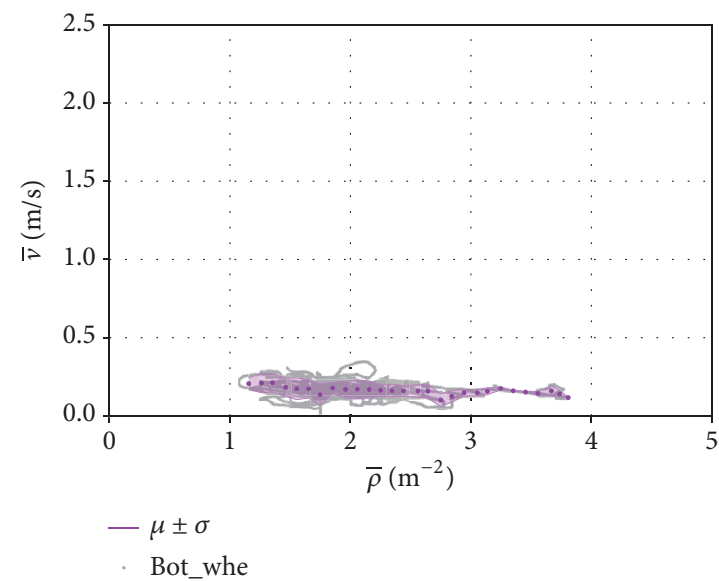

(a) Fundamental diagram $\bar{v}(\bar{\rho})$

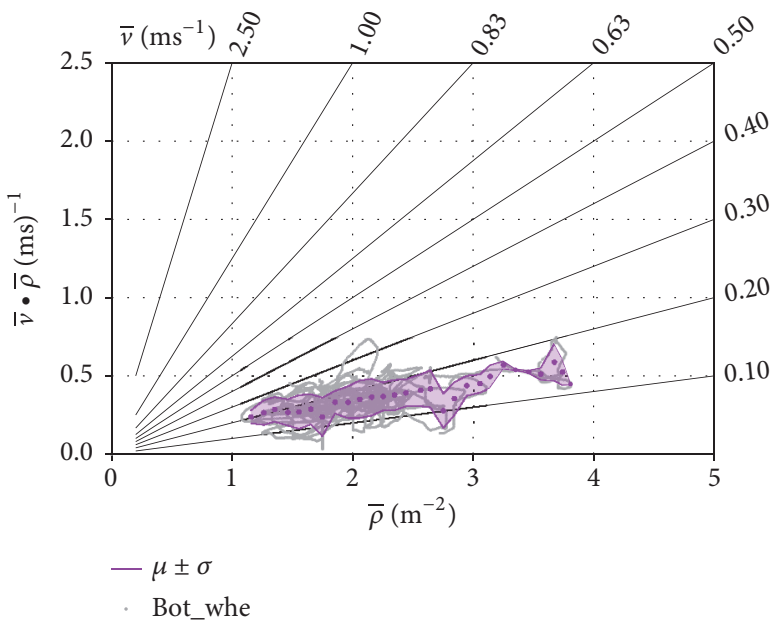

(c) Fundamental diagram $J_{s}(\bar{\rho})=\bar{\rho} \cdot \bar{v}$

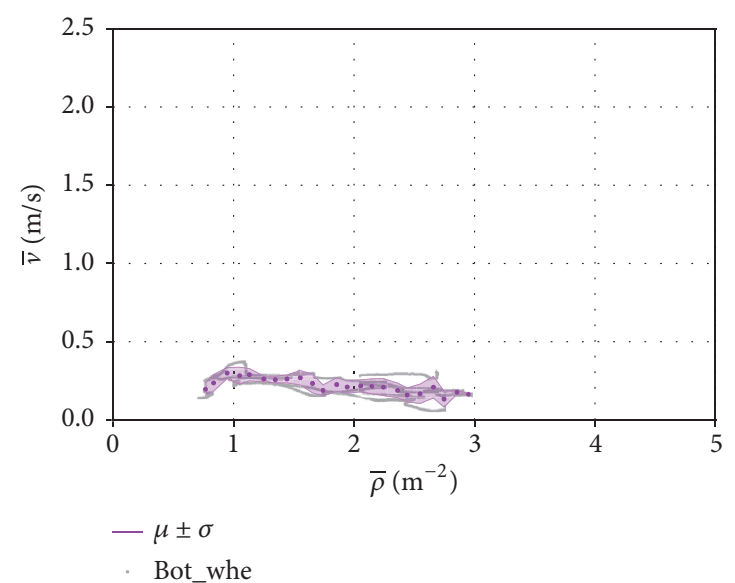

(b) Fundamental diagram $\bar{v}(\bar{\rho})$

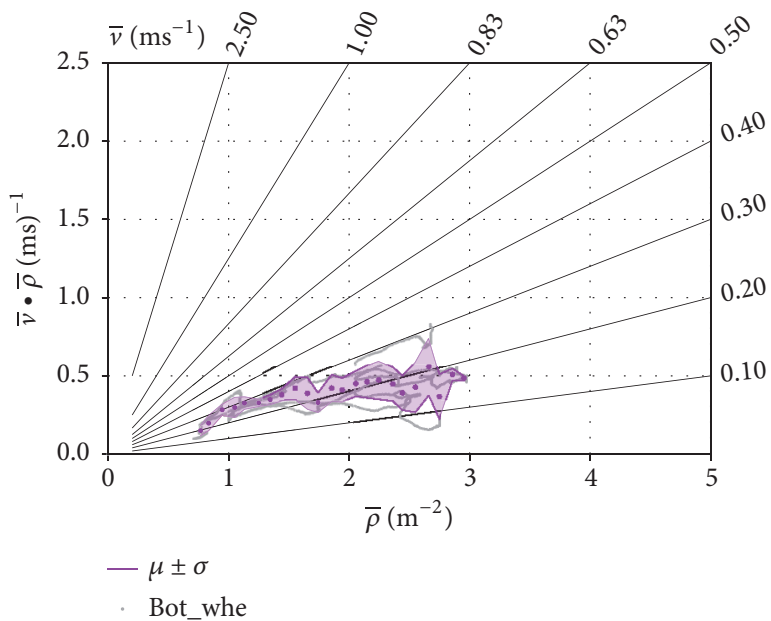

(c) Fundamental diagram $J_{s}(\bar{\rho})=\bar{\rho} \cdot \bar{v}$

FIGURE 6: Fundamental diagrams for bottleneck studies. The mean values are presented as scattered bins in density intervals of $0.1 \mathrm{~m}^{-2}$ and as mean values with standard deviation in a continuously coloured area over all runs. The point cloud coloured in grey consists of continuous data gathered from all runs. Top: fundamental diagram $\bar{v}(\bar{\rho})$ for different widths. Bottom: fundamental diagram $J_{s}(\bar{\rho})=\bar{\rho} \cdot \bar{v}$ for different widths and the grey dotted nomogram lines for associated movement speeds $v$. Left: population consists of $\approx 10 \%$ wheelchair users, right: population without wheelchair users.

that the movement speed of the slower ones determines the movement speed of the following people.

3.3. Individual Time Gap. The individual time gap for the passage of a line between a participant and the person moving ahead of him (preceding person) is analysed to quantify the individual distance headway. The headway of a pedestrian is used to maintain the distance of an individual to a predecessor because of additional space to adopt movement speed, avoid collisions, or take a step [104]. Therefore, time gaps are an important parameter to describe the movement of a crowd and it is the basis to quantify the specific flow (see Section 3.4).

The mean time gaps for the disabled and nondisabled subpopulations and the reference population in the bottleneck and a corridor situation of different widths are presented in Table 3. A noticeable difference between the time gaps of the disabled and the nondisabled subpopulations in both geometrical settings is remarkable. Because of the spatial boundary, wheelchair users tend to be slower and increase the distance to the predecessors over time. This leads to higher time gaps between the passage of a participant without disabilities and a wheelchair user (see the orange scatter in Figure 8(a)). Whilst the time gap for participants without disabilities depends on the width (for both geometries) and decreases with increasing width, the time gap of the participants in wheelchairs is constant. In our studies, the width was expanded in steps of $0.1 \mathrm{~m}$. Assuming that a wheelchair user has a minimum width of $0.8 \mathrm{~m}$, the additional cross-section has no effect for a faster movement (through the corridor).

In case of a bottleneck situation, an increased interaction and communication between participants in wheelchairs and nondisabled participants was noticed. Even if a wheelchair user reaches the entrance to the bottleneck, their neighbours anticipate individual movement speed and stop passing. They 


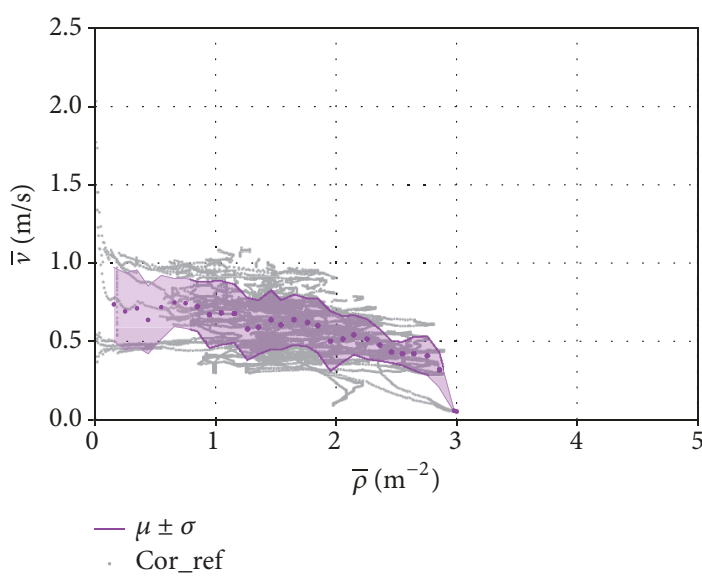

(a) Fundamental diagram $\bar{v}(\bar{\rho})$

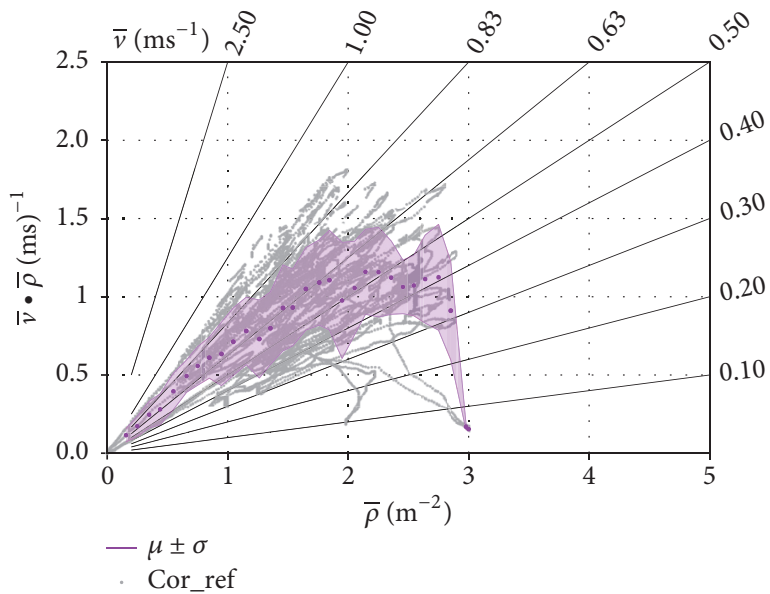

(c) Fundamental diagram $J_{s}(\bar{\rho})=\bar{\rho} \cdot \bar{v}$

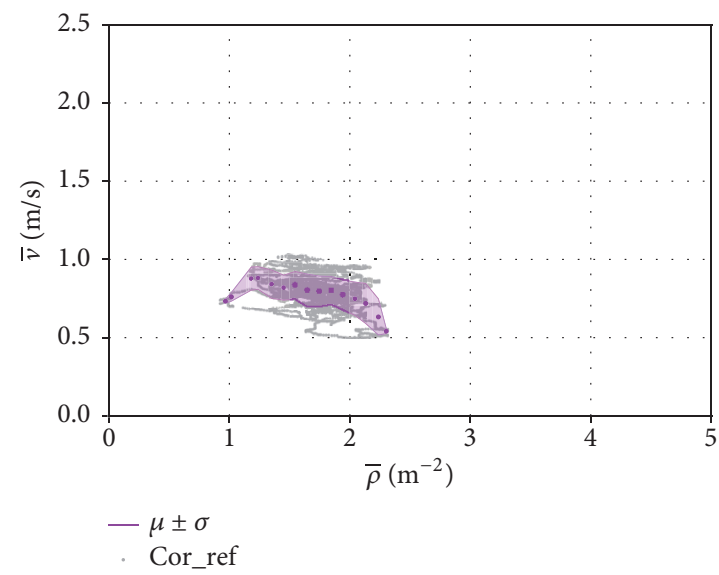

(b) Fundamental diagram $\bar{v}(\bar{\rho})$

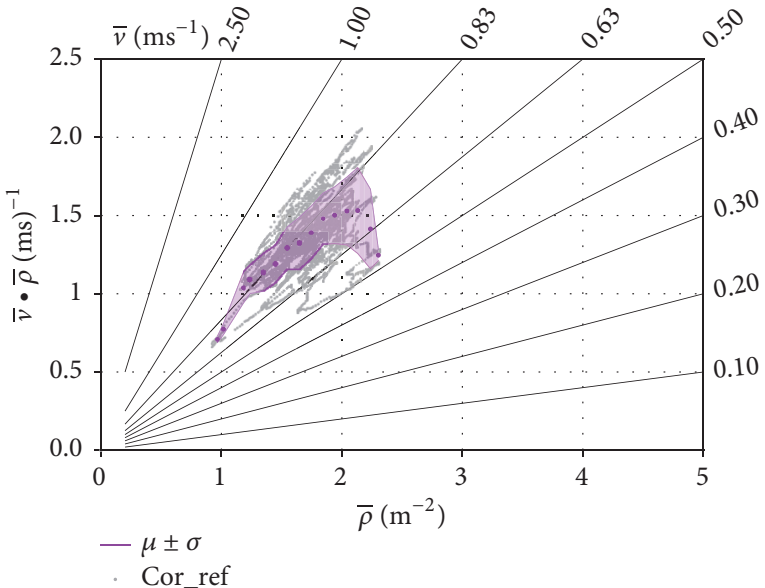

(c) Fundamental diagram $J_{s}(\bar{\rho})=\bar{\rho} \cdot \bar{v}$

Figure 7: Fundamental diagrams for corridor studies. The mean values are presented as scattered bins in density intervals of $0.1 \mathrm{~m}^{-2}$ and as mean values with standard deviation in a continuously coloured area over all runs. The point cloud coloured in grey consists of continuous data gathered from all runs. Top: fundamental diagram $\bar{v}(\bar{\rho})$ for different widths. Bottom: fundamental diagram $J_{s}(\bar{\rho})=\bar{\rho} \cdot \bar{v}$ for different widths and the grey dotted nomogram lines for associated movement speeds $v$. Left: population consists of $\approx 10 \%$ wheelchair users, right: population without wheelchair users.

interact and solve priority of movement by communication. Social norms, individual behaviour, and degrees of freedom in movement and the influence of technical assistance devices and accompanying persons may affect the passageway [105]. As a result, the time between passage of a wheelchair user and his predecessors increases.

3.4. Validity of Specific Flow Concept. One of the most important questions while assessing the performance of a facility is to quantify the capacity of a bottleneck and how the capacity increases with the width [106]. According to a common approach (method (b)) the flow here is obtained for a cumulative number of passed participants over a passage line where the time gaps were measured. The flow rate is then the derivative in time of the cumulative flow [57, p. 4] and the specific flow is the flow per unit width. The specific flow for the subpopulations wheelchair users and nondisabled participants for the bottleneck and the corridor settings is presented in Figures 9 and 10. We found high flows for studies without a disabled subpopulation which is in accordance with previous finding (e.g., [26]). As indicated by the strong difference in time gap analysis, the specific flow of the subpopulation in wheelchairs is much less than the specific flow of the nondisabled participants (Figures 9(a) and $10(\mathrm{a}))$.

It is generally assumed that the specific flow is a linear (e.g., [29, 37, 38]) or a stepping function (e.g., [28]) of the width. This behaviour was reproduced in the presented studies with the reference population but not in the case of a subpopulation using a wheelchair (compare the difference between the left and the right in Figures 9 and 10). The presence of wheelchair users has a noticeable impact: the specific flow (green dots) for the cumulative population (wheelchair users and participants without disabilities) is nearly constant in both geometries. Due to the high space requirements of wheelchair users the extended space for additional widths of 
TABLE 3: Individual passage time gap (mean + standard deviation) in $\mathrm{s}^{-1}$ for disabled and non-disabled participants in heterogeneous and homogeneous crowds.

\begin{tabular}{|c|c|c|c|c|}
\hline & \multirow[t]{2}{*}{ Run } & \multirow[t]{2}{*}{ width / m } & \multicolumn{2}{|c|}{ Time gap $\Delta t_{i} / \mathrm{s}^{-1}$} \\
\hline & & & disabled population & non-disabled population \\
\hline \multirow{2}{*}{ Bot_whe } & 01 & 0.9 & $2.51 \pm 0.88$ & $0.93 \pm 0.44$ \\
\hline & 02 & 0.9 & $2.83 \pm 1.00$ & $0.97 \pm 0.54$ \\
\hline \multirow{2}{*}{ Bot_whe } & 03 & 1.0 & $2.69 \pm 1.08$ & $0.84 \pm 0.51$ \\
\hline & 04 & 1.0 & $2.38 \pm 1.08$ & $0.74 \pm 0.40$ \\
\hline \multirow{2}{*}{ Bot_whe } & 05 & 1.1 & $2.45 \pm 0.88$ & $0.74 \pm 0.45$ \\
\hline & 06 & 1.1 & $2.75 \pm 0.73$ & $0.68 \pm 0.36$ \\
\hline \multirow{2}{*}{ Bot_whe } & 07 & 1.2 & $1.39 \pm 0.80$ & $0.77 \pm 0.68$ \\
\hline & 08 & 1.2 & $2.23 \pm 0.45$ & $0.62 \pm 0.36$ \\
\hline \multirow{2}{*}{ Bot_ref } & 01 & 0.9 & - & $0.77 \pm 0.31$ \\
\hline & 02 & 0.9 & - & $0.76 \pm 0.34$ \\
\hline \multirow{2}{*}{ Bot_ref } & 03 & 1.0 & - & $0.69 \pm 0.34$ \\
\hline & 04 & 1.0 & - & $0.67 \pm 0.39$ \\
\hline \multirow{2}{*}{ Bot_ref } & 05 & 1.1 & - & $0.61 \pm 0.40$ \\
\hline & 06 & 1.1 & - & $0.62 \pm 0.31$ \\
\hline \multirow{2}{*}{ Bot_ref } & 07 & 1.2 & - & $0.56 \pm 0.40$ \\
\hline & 08 & 1.2 & - & $0.54 \pm 0.37$ \\
\hline \multirow{2}{*}{ Cor_whe } & 01 & 0.9 & $5.21 \pm 6.87$ & $1.07 \pm 0.46$ \\
\hline & 02 & 0.9 & $3.03 \pm 2.26$ & $0.99 \pm 0.49$ \\
\hline \multirow{2}{*}{ Cor_whe } & 03 & 1.0 & $4.47 \pm 5.24$ & $0.91 \pm 0.51$ \\
\hline & 04 & 1.0 & $3.76 \pm 2.67$ & $0.87 \pm 0.40$ \\
\hline \multirow{2}{*}{ Cor_whe } & 05 & 1.1 & $3.27 \pm 3.24$ & $0.79 \pm 0.42$ \\
\hline & 06 & 1.1 & $3.00 \pm 3.26$ & $0.79 \pm 0.41$ \\
\hline \multirow{2}{*}{ Cor_whe } & 07 & 1.2 & $3.51 \pm 4.21$ & $0.77 \pm 0.40$ \\
\hline & 08 & 1.2 & $3.10 \pm 2.54$ & $0.76 \pm 0.47$ \\
\hline \multirow{2}{*}{ Cor_ref } & 01 & 0.9 & - & $0.95 \pm 0.38$ \\
\hline & 02 & 0.9 & - & $0.87 \pm 0.33$ \\
\hline \multirow{2}{*}{ Cor_ref } & 03 & 1.0 & - & $0.75 \pm 0.31$ \\
\hline & 04 & 1.0 & - & $0.72 \pm 0.31$ \\
\hline \multirow{2}{*}{ Cor_ref } & 05 & 1.1 & - & $0.72 \pm 0.43$ \\
\hline & 06 & 1.1 & - & $0.69 \pm 0.39$ \\
\hline \multirow{2}{*}{ Cor_ref } & 07 & 1.2 & - & $0.63 \pm 0.36$ \\
\hline & 08 & 1.2 & - & $0.59 \pm 0.29$ \\
\hline
\end{tabular}

$0.1 \mathrm{~m}$ to $0.3 \mathrm{~m}$ is only usable for agile, flexible participants without disabilities. This leads to no remarkable change in flow when wheelchair users are attending.

\section{Conclusions and Outlook}

A series of well-controlled studies on movement through a bottleneck and a corridor was performed. We compared the influence of different populations (with and without wheelchair users) on movement characteristics. Individual trajectories of $\approx 80$ participants per run were used for calculation; $\approx 10 \%$ of the heterogeneous crowds were participants in wheelchairs. The width of both geometries has been varied to archive different densities and to investigate the possibilities of overtaking actions.
Unimpeded movement speed of all participants was measured and analysed with respect to the individual abilities. Expected unimpeded movement speeds $\left(1.47 \pm 0.17 \mathrm{~ms}^{-1}\right)$ were observed with the nondisabled reference population which is consistent with the literature [65]. Even slower unimpeded movement speeds were observed for the disabled populations, especially for the wheelchair users $(0.96 \pm$ $\left.0.35 \mathrm{~ms}^{-1}\right)$ and the mixed population $\left(0.71 \pm 0.26 \mathrm{~ms}^{-1}\right)$.

Further on, the speed-density and flow-density relation (fundamental diagram) was studied. It was found that the basic shape of the flow-density relation is similar to previous research, but the shape of the speed-density relation is different in case of the bottleneck configuration. Because of a polite and considerate (social) behaviour, participants anticipate their movement speed to the slower wheelchair users and 


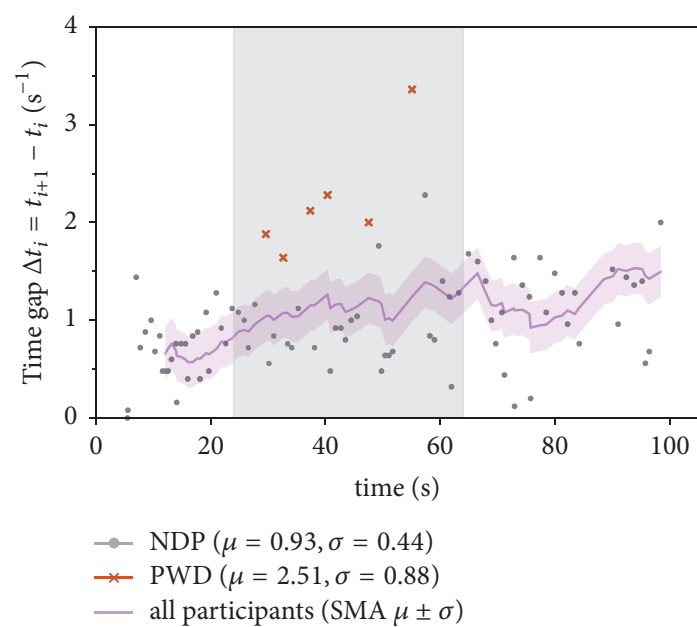

(a) Individual time gaps for run Bot_whe_01 with $\approx 10 \%$ participants using wheelchairs

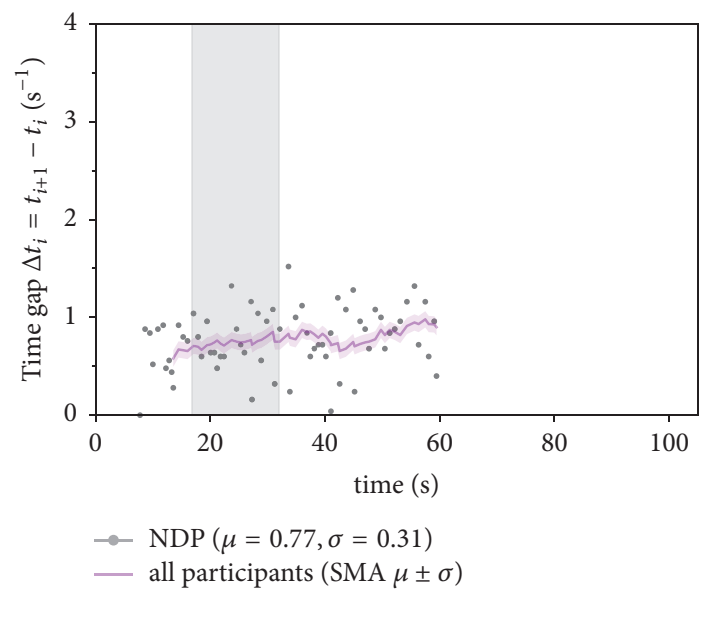

(b) Individual time gaps for run Bot_ref_01 without participants using wheelchairs

Figure 8: Comparison of passage time gaps for bottleneck studies (width $w=0.9 \mathrm{~m}$ ) considering different subpopulations.

renounced overtaking actions. This results in density regions of the fundamental diagram of $3.0 \mathrm{~m}^{-2}$ where the maximum flow is not reached.

It is found that the participants using wheelchairs keep larger distances to their predecessors, which is caused by the slower movement speeds in corridors and as a consequence of communication process in front of the bottleneck. Furthermore, additional space in case of larger widths has no effects on the time gaps of wheelchair users because the additional space is to leak for overtaking or passing the line simultaneously.

As a consequence, the specific flow concept is only approved for the reference study without disabled participants. Because of significantly lower specific flow for disabled subpopulations, the cumulative specific flow of heterogeneous group is constant and depend not on the width. It is worth mentioning that the number of data for the wheelchair users is limited and that usage of larger steps of additional widths (e.g., a multiple of a wheelchair width) may have an impact on the flow.

As a first step, the presented results may be used to improve the capacity calculations for different configurations of populations in design and planning process of facilities. Further research, especially on the comparison between different process in movement and different ratios of populations, is required.

\section{Abbreviations}

A: $\quad$ Measurement surface $/ \mathrm{m}^{2}$

Bot: Bottleneck

Cor: Corridor

$e_{i}$ : $\quad$ Effective length of the covered distance during the sampling interval $\left[t_{0}, t_{1}\right] / \mathrm{m}$
J: $\quad$ Flow rate $/ \mathrm{s}^{-1}$

$J_{s}: \quad$ Specific flow $/ \mathrm{m}^{-1} \mathrm{~s}^{-1}$

$J_{s, \max }$ : Capacity of a facility $/ \mathrm{m}^{-1} \mathrm{~s}^{-1}$

$l$ : $\quad$ Length of the bottleneck / $\mathrm{m}$

MA: Measurement area

$N$ : Numbers of participants

persID: Unique identification number of a participant

ref: $\quad$ Study with a reference population without any disabled participants

SiME: Safety for pedestrians with disabilities

$t: \quad$ Time / s

$t_{0}, t_{1}$ : Lower and upper limit of the time interval / s

$v: \quad$ Movement speed $/ \mathrm{ms}^{-1}$

$v_{0}$ : Unimpeded (free) movement speed $/ \mathrm{ms}^{-1}$

$v_{\text {jam }}$ : Propagation speed of a congestion $/ \mathrm{ms}^{-1}$

$v_{v}: \quad$ Voronoi movement speed $/ \mathrm{ms}^{-1}$

$\bar{v}: \quad$ Mean movement speed $/ \mathrm{ms}^{-1}$

$w: \quad$ Width / $\mathrm{m}$

whe: Study with a subpopulation using a wheelchair

$x$ : Horizontal axis in the Cartesian coordinate system / $\mathrm{m}$

$y$ : $\quad$ Vertical axis in the Cartesian coordinate system / m

$\mu$ : $\quad$ Mean value

$\Delta t_{i}: \quad$ Sampling interval / $\mathrm{s}$

$\rho_{c}$ : $\quad$ Density where the flow reaches the maximum / $\mathrm{m}^{-2}$

$\rho_{v}: \quad$ Voronoi density $/ \mathrm{m}^{-2}$

$\bar{\rho}: \quad$ Mean density $/ \mathrm{m}^{-2}$

$\rho_{0}$ : $\quad$ Density, where the velocity is close to zero due to overcrowding $/ \mathrm{m}^{-2}$

$\sigma: \quad$ Standard deviation 


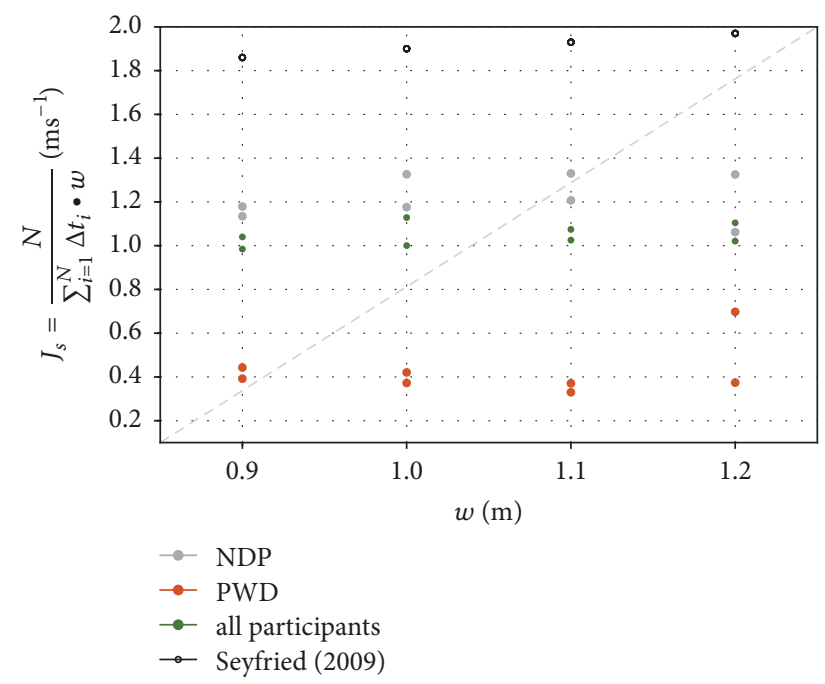

(a) Specific flow for study Bot_whe with $\approx 10 \%$ participants using wheelchairs

FIGURE 9: Comparison of specific flows for bottleneck studies considering different subpopulations in a bottleneck. Reference data is obtained from [37].

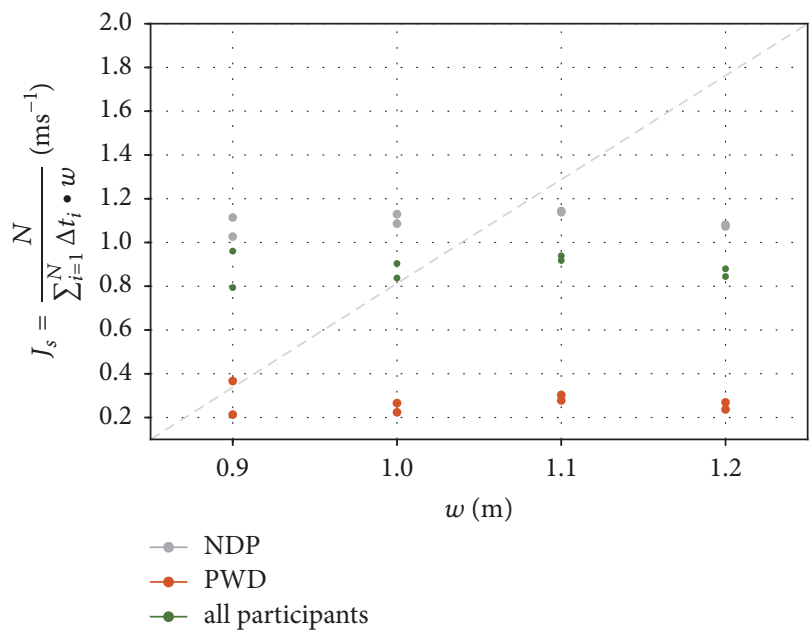

(a) Specific flow for study Cor_whe with $\approx 10 \%$ participants using wheelchairs

FIGURE 10: Comparison of specific flows for studies considering different subpopulations in a corridor. Reference data is obtained from [37].

\section{Data Availability}

Trajectory data will be published with DOI at the Juelich database about data in pedestrian dynamics (http://ped.fzjuelich.de/db/) with the latest six months after publication of this research article.

\section{Conflicts of Interest}

The authors declare that they have no conflicts of interest.

\section{Authors' Contributions}

Paul Geoerg, Jette Schumann, Stefan Holl, and Anja Hofmann carried out the problem identification and developed

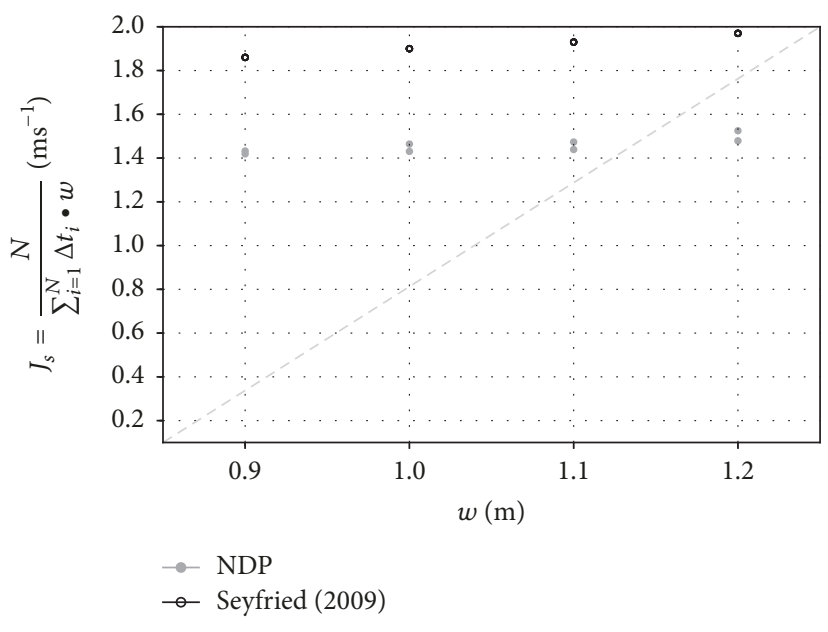

(b) Specific flow for study Bot_ref without participants using wheelchairs

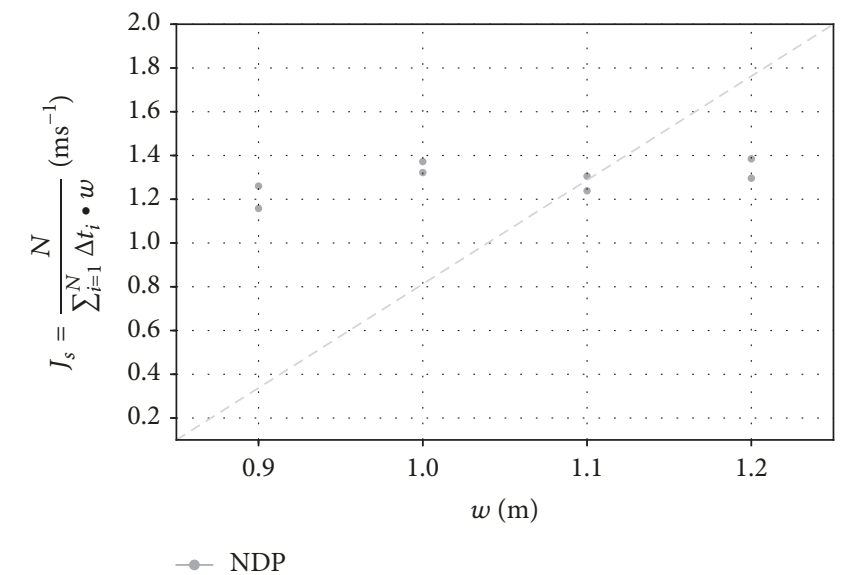

(b) Specific flow for study Cor_ref without participants using wheelchairs 
within the research program safety for people with physical, mental, or age-related disabilities (SiME) funded by the German Federal Ministry of Education and Research-BMBF (grant numbers 13N13946 and 13N13950). Paul Geoerg thanks the SFPE Foundation for financial support with Dr. Guylène Proulx, OC Scholarship.

\section{Supplementary Materials}

This section contains supplementary data for the fundamental diagrams $\bar{v}(\bar{\rho})$ and $J_{s}(\bar{\rho})=\bar{\rho} \cdot \bar{v}$ presented in Figures 6 and 7 . Here the results are presented for all runs in different widths $(w=(0.9,1.0,1.1,1.2) \mathrm{m})$ considering a population with $10 \%$ of wheelchair users in comparison to a population without participation of disabled pedestrians in a bottleneck (Figure S1) and a corridor (Figure S2). The runs are represented in different colours. According to the specific features in the boundaries, the results of the individual runs are similar and not influenced by the width of the bottleneck or corridor. (Supplementary Materials)

\section{References}

[1] S. Gwynne and K. E. Boyce, "Engineering data," in SFPE Handbook of Fire Protection Engineering, M. J. Hurley, Ed., pp. 2429-2551, Springer, New York, NY, USA, 2016.

[2] A. Seyfried, B. Steffen, W. Klingsch, and M. Boltes, "The fundamental diagram of pedestrian movement revisited," Journal of Statistical Mechanics: Theory and Experiment, vol. 2005, no. 10, Article ID P10002, pp. 41-53, 2005.

[3] G. Zeng, S. Cao, C. Liu, and W. Song, "Experimental and modeling study on relation of pedestrian step length and frequency under different headways," Physica A: Statistical Mechanics and Its Applications, vol. 500, pp. 237-248, 2018.

[4] U. Chattaraj, A. Seyfried, P. Chakroborty, and M. K. Biswal, "Modelling single file pedestrian motion across cultures," Procedia - Social and Behavioral Sciences, vol. 104, pp. 698-707, 2013.

[5] C. Feliciani and K. Nishinari, "Measurement of congestion and intrinsic risk in pedestrian crowds," Transportation Research Part C: Emerging Technologies, vol. 91, pp. 124-155, 2018.

[6] A. Jelić, C. Appert-Rolland, S. Lemercier, and J. Pettré, "Properties of pedestrians walking in line: Fundamental diagrams," Physical Review E: Statistical, Nonlinear, and Soft Matter Physics, vol. 85, no. 3, 2012.

[7] C.-J. Jin, R. Jiang, W. Wei, D. Li, and N. Guo, "Microscopic events under high-density condition in uni-directional pedestrian flow experiment," Physica A: Statistical Mechanics and its Applications, vol. 506, pp. 237-247, 2018.

[8] X. Liu, W. Song, and J. Zhang, "Extraction and quantitative analysis of microscopic evacuation characteristics based on digital image processing," Physica A: Statistical Mechanics and Its Applications, vol. 388, no. 13, pp. 2717-2726, 2009.

[9] J. Zhang, W. Mehner, S. Holl et al., "Universal flow-density relation of single-file bicycle, pedestrian and car motion," Physics Letters A, vol. 378, no. 44, pp. 3274-3277, 2014.

[10] J. Zhang, A. Tordeux, and A. Seyfried, "Effects of boundary conditions on single-file pedestrian flow," in Proceedings of the 9th International Conference on Cellular Automata, ACRI 2014, J. Wąs, G. C. Sirakoulis, and S. Bandini, Eds., vol. 8751, pp. 462469, Springer International Publishing, Cham, Switzerland, 2015.
[11] Y. Ma, Y. Y. Sun, E. W. Lee, and R. K. Yuen, "Pedestrian stepping dynamics in single-file movement," Physical Review E: Statistical, Nonlinear, and Soft Matter Physics, vol. 98, no. 6, 2018.

[12] T. Kretz, A. Grünebohm, M. Kaufman, F. Mazur, and M. Schreckenberg, "Experimental study of pedestrian counterflow in a corridor," Journal of Statistical Mechanics: Theory and Experiment, vol. 2006, no. 10, Article ID P10001, 2006.

[13] S. Holl, Methoden für die Bemessung der Leistungsfähigkeit multidirektional genutzter Fußverkehrsanlagen [Dissertation], Bergische Universität, Wuppertal, Germany, 2016.

[14] J. Y. S. Lee and W. H. K. Lam, "Variation of walking speeds on a unidirectional walkway and on a bidirectional stairway," Transportation Research Record: Journal of the Transportation Research Board, vol. 1982, pp. 122-131, 2006.

[15] X.-D. Liu, W.-G. Song, and W. Lv, "Empirical data for pedestrian counterflow through bottlenecks in the channel," Transportation Research Procedia, vol. 2, pp. 34-42, 2014.

[16] R. Nagai, T. Nagatani, M. Isobe, and T. Adachi, "Effect of exit configuration on evacuation of a room without visibility," Physica A: Statistical Mechanics and its Applications, vol. 343, no. 1-4, pp. 712-724, 2004.

[17] M. Saberi, K. Aghabayk, and A. Sobhani, "Spatial fluctuations of pedestrian velocities in bidirectional streams: Exploring the effects of self-organization," Physica A: Statistical Mechanics and its Applications, vol. 434, pp. 120-128, 2015.

[18] W. Daamen and S. P. Hoogendoorn, "Controlled experiments to derive walking behaviour," European Journal of Transport and Infrastructure Research EJTIR, vol. 3, no. 1, pp. 56-62, 1995.

[19] A. Seyfried, M. Boltes, J. Kähler et al., "Enhanced empirical data for the fundamental diagram and the flow through bottlenecks," in Pedestrian and Evacuation Dynamics, W. Klingsch, C. Rogsch, A. Schadschneider, and M. Schreckenberg, Eds., pp. 145-156, Springer, Berlin, Germany, 2010.

[20] W. Song, W. Lv, and Z. Fang, "Experiment and modeling of microscopic movement characteristic of pedestrians," Procedia Engineering, vol. 62, pp. 56-70, 2013.

[21] A. Winkens, W. Klingsch, and A. Seyfried, "New data for human performance in planar corridors," in Pedestrian and Evacuation Dynamics, R. D. Peacock, E. D. Kuligowski, and J. D. Averill, Eds., pp. 61-70, Springer, New York, NY, USA, 2011.

[22] J. Zhang, W. Klingsch, A. Schadschneider, and A. Seyfried, "Transitions in pedestrian fundamental diagrams of straight corridors and T-junctions," Journal of Statistical Mechanics: Theory and Experiment, vol. 2011, no. 6, Article ID P06004, 2011.

[23] J. Adrian, M. Boltes, S. Holl, A. Sieben, and A. Seyfried, "Crowding and queuing in entrance scenarios: influence of corridor width in front of bottlenecks," in Proceedings of the 9th Conference on Pedestrian and Evacuation Dynamics, A. S. Dederichs, Ed., pp. 77/1-77/8, Lund, Sweden, 2018.

[24] W. Daamen and S. P. Hoogendoorn, "Flow-density relations for pedestrian traffic," in Traffic and Granular Flow'05, A. Schadschneider, R. Kühne, T. Pöschel, M. Schreckenberg, and D. E. Wolf, Eds., pp. 315-322, Springer-Verlag, Berlin, Germany, 2007.

[25] W. Daamen and S. P. Hoogendoorn, "Emergency door capacity: influence of door width, population composition and stress level," Fire Technology, vol. 48, no. 1, pp. 55-71, 2012.

[26] W. Daamena and S. Hoogendoorn, "Capacity of doors during evacuation conditions," Procedia Engineering, vol. 3, pp. 53-66, 2010. 
[27] D. Helbing, L. Buzna, A. Johansson, and T. Werner, "Selforganized pedestrian crowd dynamics: experiments, simulations, and design solutions," Transportation Science, vol. 39, no. 1, pp. 1-24, 2005.

[28] S. P. Hoogendoorn and W. Daamen, "Pedestrian behavior at bottlenecks," Transportation Science, vol. 39, no. 2, pp. 147-159, 2005.

[29] T. Kretz, A. Grünebohm, and M. Schreckenberg, "Experimental study of pedestrian flow through a bottleneck," Journal of Statistical Mechanics: Theory and Experiment, vol. 2006, no. 10, Article ID P10014, 2006.

[30] W. Liao, A. Seyfried, J. Zhang, M. Boltes, X. Zheng, and Y. Zhao, "Experimental study on pedestrian flow through wide bottleneck," Transportation Research Procedia, vol. 2, pp. 26-33, 2014.

[31] W. Liao, A. Tordeux, A. Seyfried et al., "Measuring the steady state of pedestrian flow in bottleneck experiments," Physica A: Statistical Mechanics and Its Applications, vol. 461, pp. 248-261, 2016.

[32] J. Liddle, A. Seyfried, W. Klingsch, T. Rupprecht, A. Schadschneider, and A. Winkens, "An experimental study of pedestrian congestions: influence of bottleneck width and length," 2009, https://arxiv.org/abs/0911.4350.

[33] J. Liddle, A. Seyfried, B. Steffen et al., "Microscopic insights into pedestrian motion through a bottleneck, resolving spatial and temporal variations," 2011, https://arxiv.org/abs/1105.1532.

[34] H. Mu, S. Lo, W. Song, J. Wang, and J. Sun, "An experimental and numerical study of imbalanced door choice during an announced evacuation drill," Fire Technology, vol. 52, no. 3, pp. 801-815, 2016.

[35] A. Nicolas, S. Bouzat, and M. Kuperman, "Influence of selfish and polite behaviours on a pedestrian evacuation through a narrow exit: A quantitative characterisation," in Proceedings of the 8th International Conference on Pedestrian and Evacuation Dynamics, W. Song, J. Ma, and L. Fu, Eds., pp. 84-91, 2016.

[36] T. Rupprecht, W. Klingsch, and A. Seyfried, "Influence of geometry parameters on pedestrian flow through bottleneck," in Pedestrian and Evacuation Dynamics, R. D. Peacock, E. D. Kuligowski, and J. D. Averill, Eds., pp. 71-80, Springer, New York, NY, USA, 2011.

[37] A. Seyfried, O. Passon, B. Steffen, M. Boltes, T. Rupprecht, and W. Klingsch, "New insights into pedestrian flow through bottlenecks," Transportation Science, vol. 43, no. 3, pp. 395-406, 2009.

[38] A. Seyfried, T. Rupprecht, A. Winkens et al., "Capacity estimation for emergency exits and bottlenecks," in Proceedings of the INTERFLAM 2007, S. Grayson, Ed., Interscience Communications, 2007.

[39] A. Seyfried and A. Schadschneider, "Empirical results for pedestrian dynamics at bottlenecks," in Parallel Processing and Applied Mathematics, R. Wyrzykowski, J. Dongarra, K. Karczewski, and J. Wasniewski, Eds., vol. 6068 of Series Lecture Notes in Computer Science, pp. 575-584, Springer, Berlin, Germany, 2010.

[40] A. Seyfried, B. Steffen, A. Winkens, T. Rupprecht, M. Boltes, and W. Klingsch, "Empirical data for pedestrian flow through bottlenecks," in Traffic and Granular Flow' 07, C. AppertRolland, F. Chevoir, and P. Gondret, Eds., pp. 189-199, SpringerVerlag, 2009.

[41] W. Song, J. Zhang, and A. Seyfried, "Experimental study of pedestrian flow in the channel through bottleneck," in
Pedestrian and Evacuation Dynamics, R. D. Peacock, E. D. Kuligowski, and J. D. Averill, Eds., pp. 875-879, Springer, New York, NY, USA, 2011.

[42] W. Tian, W. Song, W. Lü, and Z. Fang, "Experiment and analysis on microscopic characteristics of pedestrian movement in building bottleneck," Science China Technological Sciences, vol. 54, no. 7, pp. 1730-1736, 2011.

[43] W. Tian, W. Song, J. Ma, Z. Fang, A. Seyfried, and J. Liddle, "Experimental study of pedestrian behaviors in a corridor based on digital image processing," Fire Safety Journal, vol. 47, pp. 8$15,2012$.

[44] S. Cao, A. Seyfried, J. Zhang, S. Holl, and W. Song, "Fundamental diagrams for multidirectional pedestrian flows," Journal of Statistical Mechanics: Theory and Experiment, vol. 2017, no. 3, Article ID 033404, 2017.

[45] N. Shiwakoti, Y. Gong, X. Shi, and Z. Ye, "Examining influence of merging architectural features on pedestrian crowd movement," Safety Science, vol. 75, pp. 15-22, 2015.

[46] J. Zhang, W. Klingsch, A. Schadschneider, and A. Seyfried, "Experimental study of pedestrian flow through a T-junction," in Traffic and Granular Flow' 11, V. V. Kozlov, A. P. Buslaev, A. S. Bugaev, M. V. Yashina, A. Schadschneider, and M. Schreckenberg, Eds., pp. 241-249, Springer, Berlin, Germany, 2013.

[47] N. W. Bode, M. Chraibi, and S. Holl, "The emergence of macroscopic interactions between intersecting pedestrian streams," Transportation Research Part B: Methodological, vol. 119, pp. 197210, 2019.

[48] S. Cao, L. Lian, M. Chen, M. Yao, W. Song, and Z. Fang, "Investigation of difference of fundamental diagrams in pedestrian flow," Physica A: Statistical Mechanics and its Applications, vol. 506, pp. 661-670, 2018.

[49] L. Lian, X. Mai, W. Song, Y. K. Kit Richard, X. Wei, and J. $\mathrm{Ma}$, "An experimental study on four-directional intersecting pedestrian flows," Journal of Statistical Mechanics: Theory and Experiment, vol. 2015, no. 8, Article ID P08024, 2015.

[50] D. Versluis, Microscopic interaction behavior between individual pedestrians [Master thesis], TU Delft, Delft, The Netherlands, 2010.

[51] S. C. Wong, W. L. Leung, S. H. Chan et al., "Bidirectional pedestrian stream model with oblique intersecting angle," Journal of Transportation Engineering, vol. 136, no. 3, pp. 234242, 2010.

[52] J. Zhang and A. Seyfried, "Comparison of intersecting pedestrian flows based on experiments," Physica A: Statistical Mechanics and Its Applications, vol. 405, pp. 316-325, 2014.

[53] J. Zhang, W. Klingsch, A. Schadschneider, and A. Seyfried, "Ordering in bidirectional pedestrian flows and its influence on the fundamental diagram," Journal of Statistical Mechanics: Theory and Experiment, vol. 2011/2012, no. 2, Article ID P02002, 2012.

[54] J. Zhang, Q. Wang, Y. Hu, S. Cao, L. Xia, and W. Song, “The effect of a directional split flow ratio on bidirectional pedestrian streams at signalized crosswalks," Journal of Statistical Mechanics: Theory and Experiment, vol. 2018, no. 7, p. 073408, 2018.

[55] L. D. Vanumu, K. R. Rao, and G. Tiwari, "Fundamental diagrams of pedestrian flow characteristics: A review," European Transport Research Review, vol. 9, no. 4, p. 1753, 2017.

[56] X. Shi, Z. Ye, N. Shiwakoti, and O. Grembek, "A state-of-theart review on empirical data collection for external governed pedestrians complex movement," Journal of Advanced Transportation, vol. 2018, Article ID 1063043, 42 pages, 2018. 
[57] M. Boltes, J. Zhang, A. Tordeux, A. Schadschneider, and A. Seyfried, "Empirical results of pedestrian and evacuation dynamics," in Complex Dynamics of Traffic Management, R. A. Meyers, Ed., Encyclopedia of Complexity and Systems Science Series (ECSSS), pp. 671-699, SpringerReference, New York, NY, USA, 2019.

[58] M. Haghani and M. Sarvi, "Crowd behaviour and motion: Empirical methods," Transportation Research Part B: Methodological, vol. 107, pp. 253-294, 2018.

[59] J. Wang, W. Weng, M. Boltes, J. Zhang, A. Tordeux, and V. Ziemer, "Step styles of pedestrians at different densities," Journal of Statistical Mechanics: Theory and Experiment, vol. 2018, no. 2, Article ID 023406, 21 pages, 2018.

[60] M. Spearpoint and H. A. MacLennan, "The effect of an ageing and less fit population on the ability of people to egress buildings," Safety Science, vol. 50, no. 8, pp. 1675-1684, 2012.

[61] K. Boyce, "Safe evacuation for all - Fact or Fantasy? Past experiences, current understanding and future challenges," Fire Safety Journal, vol. 91, pp. 28-40, 2017.

[62] United Nations, "World population prospects: the 2015 revision: comprehensive tables," 2015, http://esa.un.org/unpd/wpp/.

[63] M. Forhan and S. V. Gill, "Obesity, functional mobility and quality of life," Best Practice \& Research. Clinical Endocrinology \& Metabolism, vol. 27, no. 2, pp. 129-137, 2013.

[64] P. Thompson, D. Nilsson, K. Boyce, and D. McGrath, "Evacuation models are running out of time," Fire Safety Journal, vol. 78, pp. 251-261, 2015.

[65] P. Geoerg, S. Gwynne, K. Boyce, F. Berchtold, S. Holl, and A. Hofmann, "Engineering egress data considering pedestrians with reduced mobility: (under Review)," Fire and Materials, 2019.

[66] V. V. Kholshchevnikov, D. A. Samoshin, A. P. Parfyonenko, and I. P. Belosokhov, "Study of children evacuation from pre-school education institutions," Fire and Materials, vol. 36, no. 5-6, pp. 349-366, 2012.

[67] V. V. Kholshevnikov, D. A. Samoshin, and R. Istratov, "The problems of elderly people safe evacuation from senior citizen health care buildings in case of fire," in Human Behaviour in Fire, T. J. Shields, Ed., pp. 587-593, Interscience Communications, London, UK, 2012.

[68] A. R. Larusdottir and A. S. Dederichs, "A step towards including children's evacuation parameters and behavior in fire safe building design," Fire Safety Science, vol. 10, pp. 187-195, 2011.

[69] A. R. Larusdottir and A. S. Dederichs, "Evacuation of children: movement on stairs and on horizontal plane," Fire Technology, vol. 48, no. 1, pp. 43-53, 2012.

[70] S. Cao, J. Zhang, D. Salden, J. Ma, C. Shi, and R. Zhang, "Pedestrian dynamics in single-file movement of crowd with different age compositions," Physical Review E: Statistical, Nonlinear, and Soft Matter Physics, vol. 94, no. 1, 2016.

[71] S. Cao, J. Zhang, W. Song, C. Shi, and R. Zhang, “The stepping behavior analysis of pedestrians from different age groups via a single-file experiment," Journal of Statistical Mechanics: Theory and Experiment, vol. 2018, no. 3, p. 033402, 2018.

[72] W. Daamen and S. Hoogendoorn, "Calibration of pedestrian simulation model for emergency doors by pedestrian type," Transportation Research Record, vol. 2316, no. 1, pp. 69-75, 2012.

[73] X. Ren, J. Zhang, W. Song, and S. Cao, “The fundamental diagrams of elderly pedestrian flow in straight corridors under different densities," Journal of Statistical Mechanics: Theory and Experiment, vol. 2019, no. 2, p. 023403, 2019.
[74] J. G. Sørensen and A. S. Dederichs, "Evacuation characteristics of visually impaired people - a qualitative and quantitative study," Fire and Materials, vol. 39, no. 4, pp. 385-395, 2013.

[75] J. G. Sørensen and A. S. Dederichs, "Evacuation characteristics of visually impaired people-a qualitative and quantitative study," Fire and Materials, vol. 39, no. 4, pp. 385-395, 2015.

[76] D. A. Samoshin and R. N. Istratov, "The characteristics of blind and visually impaired people evacuation in case of fire," Fire Safety Science, vol. 11, pp. 1160-1169, 2014.

[77] S. Cao, P. Wang, M. Yao, and W. Song, "Dynamic analysis of pedestrian movement in single-file experiment under limited visibility," Communications in Nonlinear Science and Numerical Simulation, vol. 69, pp. 329-342, 2019.

[78] M. S. Sharifi, D. Stuart, K. Christensen, and A. Chen, “Traffic flow characteristics of heterogeneous pedestrian stream involving individuals with disabilities," Transportation Research Record, vol. 2537, pp. 111-125, 2015.

[79] S. Tsuchiya, Y. Hasemi, and Y. Furukawa, "Evacuation characteristics of group with wheelchair users," in Proceedings of the Asia-Oceania Symposium on Fire Science and Technology, 2007.

[80] S. Huang, R. Wei, S. Lo et al., "Experimental study on onedimensional movement of luggage-laden pedestrian," Physica A: Statistical Mechanics and its Applications, vol. 516, pp. 520$528,2019$.

[81] L. Luo, Z. Fu, X. Zhou, K. Zhu, H. Yang, and L. Yang, "Fatigue effect on phase transition of pedestrian movement: experiment and simulation study," Journal of Statistical Mechanics: Theory and Experiment, vol. 2016, no. 10, Article ID 103401, 31 pages, 2016.

[82] W. Daamen and S. P. Hoogendoorn, "Emergency door capacity: influence of population composition and stress level," in Pedestrian and Evacuation Dynamics, R. D. Peacock, E. D. Kuligowski, and J. D. Averill, Eds., pp. 15-24, Springer, New York, NY, USA, 2011.

[83] G. Zeng, A. Schadschneider, J. Zhang, S. Wei, W. Song, and R. $\mathrm{Ba}$, "Experimental study on the effect of background music on pedestrian movement at high density," Physics Letters A, vol. 383, no. 10, pp. 1011-1018, 2019.

[84] P. Geoerg, R. Block, W. Heister, S. Holl, A. Pulm, and A. Hofmann, "A score regarding the need for assistance: considering pedestrians with disabilities in evacuation planning," in Proceedings of the 5th Magdeburg Fire and Explosion Prevention, U. Krause and M. Rost, Eds., 2017.

[85] P. Geoerg, R. Block, W. Heister, S. Holl, A. Pulm, and A. Hofmann, "A score regarding the need for assistance - evacuation considering people with disabilities: (under review)," Fire Safety Journal, 2019.

[86] Statistisches Bundesamt, "Sozialleistungen: Schwerbehinderte Menschen: Fachserie 13," Reihe 5.1, Wiesbaden, Germany, 2019.

[87] Eurostat Statistics Explained, "Disability statistics - need for assistance," 2015, https://ec.europa.eu/eurostat/statistics-explained/index.php?title=Disability_statistics_-_need_for_assistance\#People_with_disabilities_requiring_assistance.

[88] M. W. Brault, "Americans with disabilities," Report Number P70-131, 2012, https://www.census.gov/library/publications/ 2012/demo/p70-131.html.

[89] C. Schäfer, R. Zinke, L. Künzer, G. Hofinger, and R. Koch, "Applying persona method for describing users of escape routes," Transportation Research Procedia, vol. 2, pp. 636-641, 2014. 
[90] Deutsches Institut für Normung, Barrierefreies Bauen - Planungsgrundlagen - Teil 1: Öffentlich zugängliche Gebäude, Berlin, Germany, 2010.

[91] M. Boltes and A. Seyfried, "Collecting pedestrian trajectories," Neurocomputing, vol. 100, pp. 127-133, 2013.

[92] B. S. Kerner, S. Holl, A. Tordeux, A. Seyfried, A. Schadschneider, and U. Lang, "Influences of extraction techniques on the quality of measured quantities of pedestrian characteristics," in Proceedings of the 8th International Conference on Pedestrian and Evacuation Dynamics, W. Song, J. Ma, and L. Fu, Eds., pp. 540-547, Springer US, 2016.

[93] B. Steffen and A. Seyfried, "Methods for measuring pedestrian density, flow, speed and direction with minimal scatter," Physica A: Statistical Mechanics and its Applications, vol. 389, no. 9, pp. 1902-1910, 2010.

[94] J. Zhang, Pedestrian Fundamental Diagrams: Comparative Analysis of Experiments in Different Geometries, Zugl.: Wuppertal, Univ., Wuppertal, Germany, 2012, ser. Schriften des Forschungszentrums Jülich IAS series.

[95] S. Holl, M. Boltes, and A. Seyfried, "Level of safety concept for major events," in Traffic and Granular Flow '15, V. Knoop and W. Daamen, Eds., Springer, New York, NY, USA, 2016.

[96] A. Kleffmann, S. Weinmann, F. Föhres, B. Müller, and F. Föhres, Psychologische Merkmalprofile zur Eingliederung Behinderter in Arbeit, Teilprojekt Psychologie, Siegen, Germany, 1997.

[97] T. Achterberg, H. Wind, P. Prinzie, and M. Frings-Dresen, "Inter-rater reliability of the 'merkmalprofile zur eingliederung leistungsgewandelter und behinderter in arbeit' (MELBA) in young disabled adults with psychosocial limitations," Work, vol. 44, no. 4, pp. 491-497, 2013.

[98] A. Tomoeda, D. Yanagisawa, T. Imamura, and K. Nishinari, "Propagation speed of a starting wave in a queue of pedestrians," Physical Review E: Statistical, Nonlinear, and Soft Matter Physics, vol. 86, no. 3, Article ID 036113, 2012.

[99] A. Tordeux and A. Schadschneider, "White and relaxed noises in optimal velocity models for pedestrian flow with stop-and-go waves," Journal of Physics A: Mathematical and General, vol. 49, no. 18, Article ID 185101, 16 pages, 2016.

[100] B. J. Schroeder, "Traffic operations," in Highway Engineering, D. J. Findley, B. J. Schroeder, C. M. Cunningham, and T. H. Brown, Eds., pp. 255-432, Elsevier Ltd, Amsterdam, Netherlands, 2016.

[101] L. Edie, "Discussion of traffic stream measurements and definitions," in Proceedings of the Second International Symposium on the Theory of Traffic Flow, A. Joyce, Ed., pp. 139-154, 1963.

[102] R. D. Kühne, Ed., Hinweise zum Fundamentaldiagramm: Grundlagen und Anwendungen, vol. 385 of ser. FGSV, 2005, ausg. 2005 ed.

[103] J. Zhang and A. Seyfried, "Quantification of bottleneck effects for different types of facilities," Transportation Research Procedia, vol. 2, pp. 51-59, 2014.

[104] S. Huang, T. Zhang, S. Lo, S. Lu, and C. Li, "Experimental study of individual and single-file pedestrian movement in narrow seat aisle," Physica A: Statistical Mechanics and its Applications, vol. 509, pp. 1023-1033, 2018.

[105] P. Geoerg, J. Schumann, M. Boltes, S. Holl, and A. Hofmann, "The influence of physical and mental constraints to a pedestrian stream through a bottleneck," in Proceedings of the 9th Conference on Pedestrian and Evacuation Dynamics, A. S. Dederichs, Ed., Lund, Sweden, 2018.

[106] A. Schadschneider and A. Seyfried, "Empirical results for pedestrian dynamics and their implications for modeling,"
Networks and Heterogeneous Media, vol. 6, no. 3, pp. 545-560, 2011. 


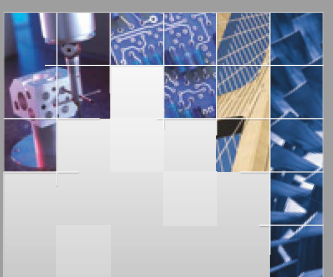

\section{Enfincering}
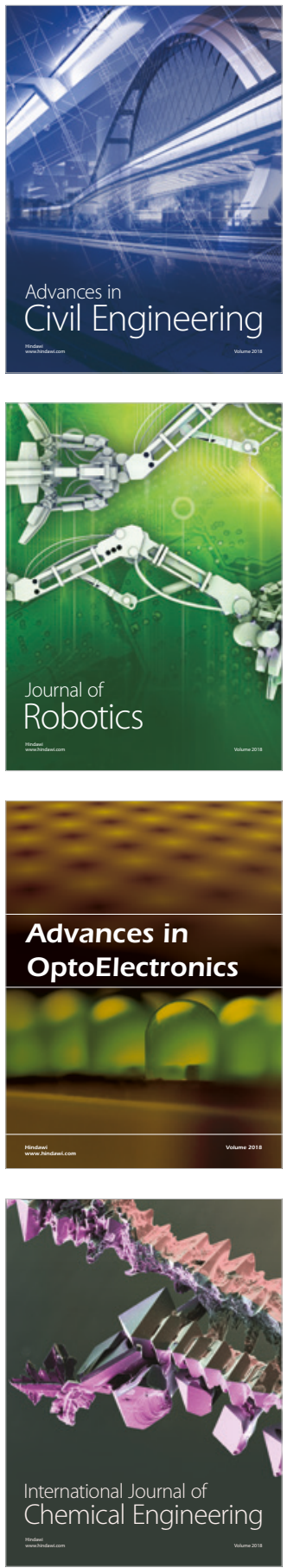

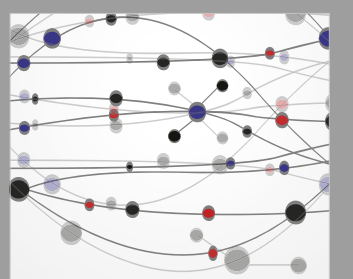

\section{Rotating \\ Machinery}

The Scientific World Journal

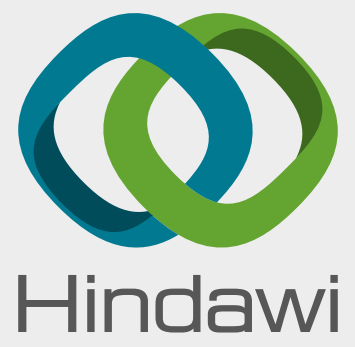

Submit your manuscripts at

www.hindawi.com
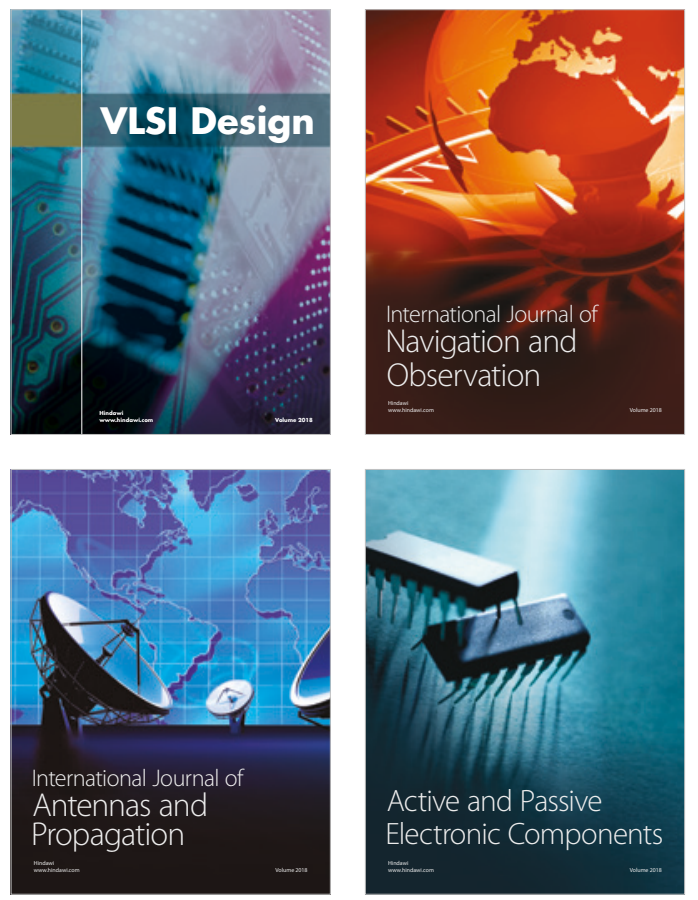
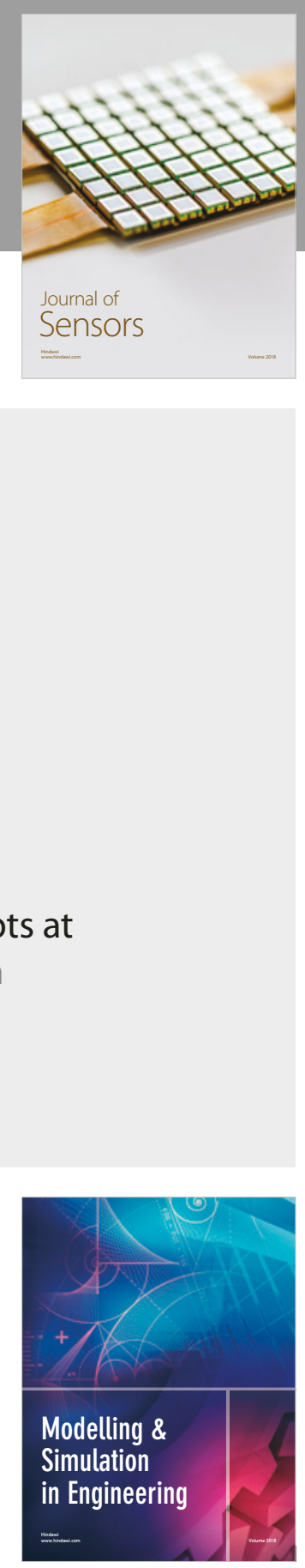

\section{Advances \\ Multimedia}
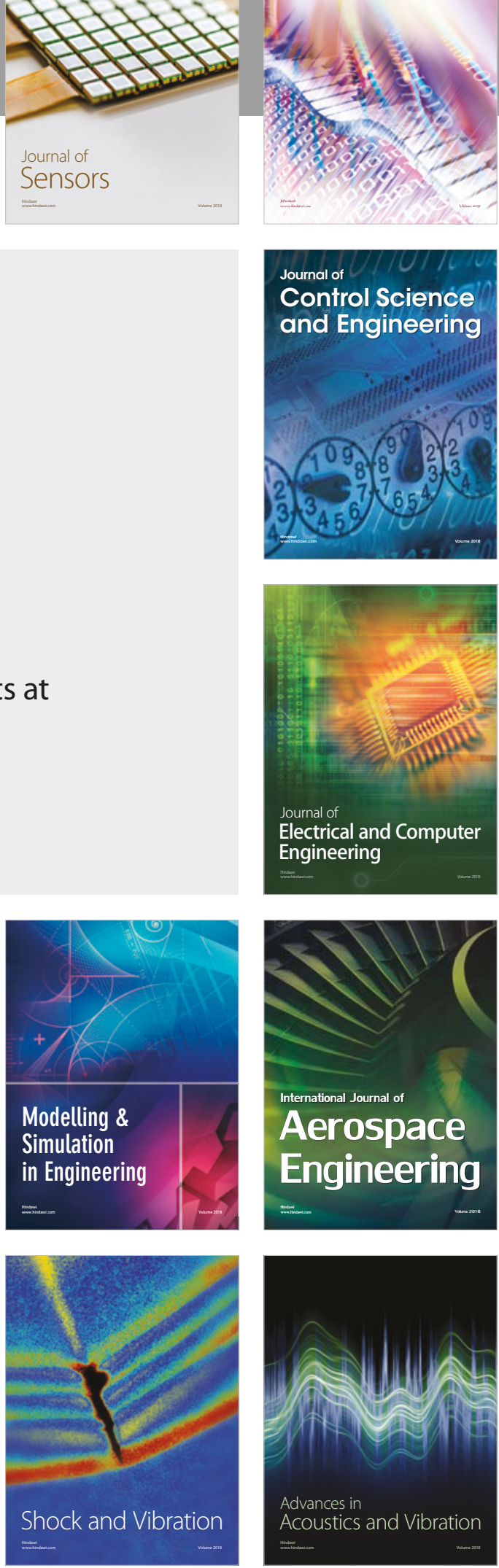